\title{
A GENERAL APPROACH FOR FRONT-PROPAGATION IN FUNCTIONAL REACTION-DIFFUSION EQUATIONS
}

\author{
ALESSANDRO CALAMAI, CRISTINA MARCELLI, AND FRANCESCA PAPALINI
}

\begin{abstract}
We investigate the persistence of front propagation for functional reaction-diffusion equations$$
v_{\tau}=v_{x x}+F(v)
$$

where $F$ is a given operator. By combining the upper and lower solution method with fixed point techniques, we prove a general existence theorem for traveling waves. Our result applies to reactiondiffusion equation with delayed or non-local reaction term.
\end{abstract}

Keywords and Phrases. Delayed reaction-diffusion equations, non-local reaction-diffusion equations, traveling waves, monostable reaction-term, upper and lower-solutions method, fixed point.

AMS Subject Classification. Primary: 35K57, 34K10; secondary: 35B40, 35R10

\section{INTRODUCTION}

The study of the existence and qualitative properties of traveling fronts for reaction-diffusion equations is a widely investigated field of research, due to several applications in various biological phenomena (see e.g. [11]).

The usual Fisher-KPP equation modeling a reaction-diffusion process is

$$
v_{\tau}=v_{x x}+f(v), \quad \tau \geq 0, x \in \mathbb{R}
$$

where $f \in C^{1}[0,1]$ satisfies $f(0)=f(1)=0, f(v)>0$ in $(0,1)$. The study of traveling wave solutions (t.w.s.) connecting the stationary states 0 and 1 has a great relevance in the investigation of the asymptotic behavior (for $\tau$ large) of a generic solution of the associated initial value problem since it is known that in certain cases the solution evolves (in some sense) towards the t.w.s. having minimal speed (see [7]). More in detail, a t.w.s. is a solution of the equation having a constant profile: $v(\tau, x)=u(x-c \tau)$ for some function $u \in C^{2}(\mathbb{R})$ (the wave profile) and constant $c$ (the wave speed). It is well-known that there exists a threshold value $c^{*}$ such that there exist fronts having speed $c^{*}$ if and only if $c \geq c^{*}$ and the stable t.w.s. corresponds to the minimal speed $c^{*}$. This value is unknown in general, but the following estimate holds:

$$
2 \sqrt{f^{\prime}(0)} \leq c^{*} \leq 2 \sqrt{\sup _{u \in(0,1]} \frac{f(u)}{u}} .
$$

Of course, when $f$ is concave, the inequalities in the previous formula are actually equalities and $c^{*}=$ $2 \sqrt{f^{\prime}(0)}$. The research in this field has been carried out also for equations having non-constant diffusivity or in the presence of a convective term. We refer to the monographs [11], [13], [2], [3] and references therein contained. 
Recently, some models of non-local reaction-diffusion equations have been proposed by S.A. Gourley (see [4]), in which the reaction term contains a convolution integral

$$
v_{\tau}=v_{x x}+v(\tau, x)\left(1-\int_{-\infty}^{+\infty} \Phi(x-y) v(\tau, y) \mathrm{d} y\right)
$$

where the kernel $\Phi$ is even, non-negative, summable in $\mathbb{R}$ with unitary integral (normalized). The classical local Fisher equation (with $f(v)=v(1-v)$ ) can be considered as a particular case, arising when the kernel $\Phi$ is the Dirac delta function.

In this setting the non-local term has the meaning of a weighted average of the density $u$ and models interactions between individuals competing not only with those localized at their own point, but also with individuals in other points of the domain. A prototype kernel is the so-called Laplace exponential distribution

$$
\Phi(\xi):=\frac{b}{2} e^{-b|\xi|}, \quad b>0
$$

naturally arising from the model of the consumption of resources. Note that for large values of $b$ the interaction is strong with close points, so the value $\frac{1}{b}$ can be considered as a measure of the non-locality (see [4]).

To the best of our knowledge, no rigorous analysis is available for such equations. Actually, in [4] an approximate model has been investigated from a qualitative point of view.

Another field having an increasing interest, is that of reaction-diffusion equations or systems with time-delay, in which the reaction term also depends on $v(\tau-T, x)$. In this area several results have been obtained, but mainly for specific forms of the reaction term (see, e.g., [1], [5], [6] and [14]). Moreover, some of the most recent ones present some problematic aspect (see Remark 4.2).

The aim of the present paper is to propose a general unifying approach for dealing with functional reaction-diffusion equations, including both the non-local equations and the delayed ones, and to enlarge the class of reaction terms to which one can apply the general existence result. More precisely, we consider the following general boundary value problem

$$
\left\{\begin{array}{l}
u^{\prime \prime}+c u^{\prime}+F(u)=0 \\
u(-\infty)=1, u(+\infty)=0
\end{array}\right.
$$

where $F: C(\mathbb{R}) \rightarrow C(\mathbb{R})$ is a given functional operator. Assuming the existence of a pair of well-ordered upper and lower-solutions, we prove a general existence result (see Theorem 4.3). Moreover, we show how it can be applied to reaction-diffusion equations having non-local or delayed terms, showing that under certain limitations on the wave speed $c$, a pair of upper and lower-solutions can be actually found (see Theorems 5.2, 5.3, 5.7).

In particular, our existence result can be fruitfully applied to delayed reaction-diffusion equation of the type

$$
v_{\tau}(\tau, x)=v_{x x}(\tau, x)+g(v(\tau, x)) v(\tau-T, x)
$$

or non-local equations of the type

$$
v_{\tau}(\tau, x)=v_{x x}(\tau, x)+g(v(\tau, x)) \int_{-\infty}^{+\infty} \Phi(x-\sigma) v(\tau, \sigma) \mathrm{d} \sigma,
$$

where $g$ is a generic Lipschitz function satisfying

$$
0<g(u) \leq g(0)(1-u) \quad \text { for every } u \in[0,1) .
$$


We underline that both in the non-local setting and in the delayed one, we obtain the existence of t.w.s. when the speed c lies in a certain interval $\left[c_{1}^{*}, c_{2}^{*}\right]$. We show that in both cases, when the model tends to the usual Fisher-KPP one, for instance when the delay tends to 0 or the kernel in the non-local equation tends to the Dirac delta function, then the left endpoint $c_{1}^{*}$ tends to the threshold value $c^{*}$ of the non-functional equation and the right endpoint $c_{2}^{*}$ diverges to $+\infty$.

Our approach is based on fixed point techniques combined to the method of upper and lower-solutions, following an idea considered by S. Ma in [8] (see Section 2). In Sections 3 and 4 we present general existence results, which are applied to non-local or delayed equations in Section 5.

\section{AN AUXILIARY PROBLEM}

This section is devoted to some preliminary results related to an auxiliary linear problem.

Let $c \in \mathbb{R}, \beta>0$ be fixed. Given $h \in L^{\infty}(\mathbb{R})$, in what follows we will consider the function $u_{h}: \mathbb{R} \rightarrow \mathbb{R}$ defined by

$$
u_{h}(t):=-e^{\alpha_{1} t} \int_{-\infty}^{t} h(s) e^{-\alpha_{1} s} \mathrm{~d} s-e^{\alpha_{2} t} \int_{t}^{+\infty} h(s) e^{-\alpha_{2} s} \mathrm{~d} s, \quad t \in \mathbb{R},
$$

where $\alpha_{1}<0<\alpha_{2}$ are the solutions of the algebraic equation $x^{2}+c x-\beta=0$.

The following result concerns some properties of the function $u_{h}$.

Lemma 2.1. Let $h \in L^{\infty}(\mathbb{R})$. Then

i) $u_{h}$ is a $C^{1}$-function on $\mathbb{R}$, with $u_{h}^{\prime}$ a.e. derivable, and

$$
u_{h}^{\prime \prime}(t)+c u_{h}^{\prime}(t)-\beta u_{h}(t)=\left(\alpha_{2}-\alpha_{1}\right) h(t) \quad \text { for a.e. } t \in R
$$

ii) if $h(t) \leq 0$ for a.e. $t \in \mathbb{R}$, then $u_{h}(t) \geq 0$ for every $t \in \mathbb{R}$.

iii) if $-\beta \leq h(t) \leq 0$ for a.e. $t \in \mathbb{R}$, then $0 \leq u_{h}(t) \leq \alpha_{2}-\alpha_{1}$ and $\left|u_{h}^{\prime}(t)\right| \leq 2 \beta$ for every $t \in \mathbb{R}$;

iv) if $h$ monotone increasing, then $u_{h}$ is monotone decreasing.

$v)$ if $h$ is continuous for $|t|$ large and if $h( \pm \infty)$ exists, then $u_{h}( \pm \infty)$ exists too and $u_{h}( \pm \infty)=$ $-h( \pm \infty) \frac{\alpha_{2}-\alpha_{1}}{\beta}$.

Proof. First observe that $u_{h}$ is absolutely continuous on every compact interval of $\mathbb{R}$, hence it is derivable for a.e. $t \in \mathbb{R}$ with

$$
u_{h}^{\prime}(t)=-\alpha_{1} e^{\alpha_{1} t} \int_{-\infty}^{t} h(s) e^{-\alpha_{1} s} \mathrm{~d} s-\alpha_{2} e^{\alpha_{2} t} \int_{t}^{+\infty} h(s) e^{-\alpha_{2} s} \mathrm{~d} s \quad \text { a.e. } t \in \mathbb{R} .
$$

The right-hand side of (2.2) is a continuous function on $\mathbb{R}$, call it $\gamma(t)$. So, since $u_{h}$ is absolutely continuous in every compact interval, fixed $t \in \mathbb{R}$ we have $u_{h}(t)=u_{h}(0)+\int_{0}^{t} u_{h}^{\prime}(s) \mathrm{d} s=u_{h}(0)+\int_{0}^{t} \gamma(s) \mathrm{d} s$ hence $u_{h} \in C^{1}(\mathbb{R})$ and $(2.2)$ holds for every $t \in \mathbb{R}$. Therefore, $u_{h}^{\prime}$ is absolutely continuous in every compact interval and

$$
u_{h}^{\prime \prime}(t)=-\alpha_{1}^{2} e^{\alpha_{1} t} \int_{-\infty}^{t} h(s) e^{-\alpha_{1} s} \mathrm{~d} s-\alpha_{2}^{2} e^{\alpha_{2} t} \int_{t}^{+\infty} h(s) e^{-\alpha_{2} s} \mathrm{~d} s+\left(\alpha_{2}-\alpha_{1}\right) h(t) \quad \text { a.e. } t \in \mathbb{R} .
$$

Recalling that $\alpha_{1}$ and $\alpha_{2}$ satisfy the equation $x^{2}+c x-\beta=0$, one gets $u_{h}^{\prime \prime}(t)+c u_{h}^{\prime}(t)-\beta u_{h}(t)=$ $\left(\alpha_{2}-\alpha_{1}\right) h(t)$ for a.e. $t \in \mathbb{R}$, and assertion $\left.i\right)$ is proved.

Property $i$ ) is immediate. Let us prove $i i i)$. 
If $h(t) \geq-\beta$ for a.e. $t \in \mathbb{R}$, then for every $t \in \mathbb{R}$ we have

$$
u_{h}(t) \leq \beta\left(e^{\alpha_{1} t} \int_{-\infty}^{t} e^{-\alpha_{1} s} \mathrm{~d} s+e^{\alpha_{2} t} \int_{t}^{+\infty} e^{-\alpha_{2} s} \mathrm{~d} s\right)=\beta\left(-\frac{1}{\alpha_{1}}+\frac{1}{\alpha_{2}}\right)=\alpha_{2}-\alpha_{1}
$$

(recall that $\alpha_{1} \alpha_{2}=-\beta$ ).

Furthermore, if $-\beta \leq h(t) \leq 0$ for a.e. $t \in \mathbb{R}$, recalling that $\alpha_{1}<0<\alpha_{2}$, we have

$$
\left|u_{h}^{\prime}(t)\right| \leq \beta\left(\alpha_{2} e^{\alpha_{2} t} \int_{t}^{+\infty} e^{-\alpha_{2} s} \mathrm{~d} s-\alpha_{1} e^{\alpha_{1} t} \int_{-\infty}^{t} e^{-\alpha_{1} s} \mathrm{~d} s\right)=2 \beta \quad \text { for every } t \in \mathbb{R} .
$$

As for property $i v$ ), let us fix $T>0$ and observe that

$$
\begin{aligned}
u_{h}(t+T) & =-e^{\alpha_{1} t} \int_{-\infty}^{t+T} h(s) e^{-\alpha_{1}(s-T)} \mathrm{d} s-e^{\alpha_{2} t} \int_{t+T}^{+\infty} h(s) e^{-\alpha_{2}(s-T)} \mathrm{d} s \\
& =-e^{\alpha_{1} t} \int_{-\infty}^{t} h(\sigma+T) e^{-\alpha_{1} \sigma} d \sigma-e^{\alpha_{2} t} \int_{t}^{+\infty} h(\sigma+T) e^{-\alpha_{2} \sigma} d \sigma .
\end{aligned}
$$

with $\sigma=s-T$. Thus,

$$
u_{h}(t+T)-u_{h}(t)=-e^{\alpha_{1} t} \int_{-\infty}^{t}(h(s+T)-h(s)) e^{-\alpha_{1} s} \mathrm{~d} s-e^{\alpha_{2} t} \int_{t}^{+\infty}(h(s+T)-h(s)) e^{-\alpha_{2} s} \mathrm{~d} s .
$$

Hence, if $h$ is increasing, then $h(s+T)-h(s) \geq 0$ a.e. $s \in \mathbb{R}$ and consequently $u_{h}(t+T)-u_{h}(t) \leq 0$ for any $t \in \mathbb{R}$, i.e. $u_{h}$ is decreasing.

Finally, let us prove $v$ ). By the L'Hopital rule,

$$
\lim _{t \rightarrow \pm \infty} u_{h}(t)=\lim _{t \rightarrow \pm \infty}\left(\frac{h(t) e^{-\alpha_{1} t}}{\alpha_{1} e^{-\alpha_{1} t}}-\frac{h(t) e^{-\alpha_{2} t}}{\alpha_{2} e^{-\alpha_{2} t}}\right)=h( \pm \infty)\left(\frac{1}{\alpha_{1}}-\frac{1}{\alpha_{2}}\right)=h( \pm \infty) \frac{\alpha_{2}-\alpha_{1}}{-\beta} .
$$

Let $B C(\mathbb{R})$ denote the space of all the bounded continuous maps $u: \mathbb{R} \rightarrow \mathbb{R}$. For every $\rho>0$, we can introduce a norm $\|\cdot\|_{\rho}$ in the space $B C(\mathbb{R})$ by defining

$$
\|u\|_{\rho}:=\sup _{t \in \mathbb{R}}|u(t)| e^{-\rho|t|}<+\infty .
$$

From now on, $B C_{\rho}(\mathbb{R})$ will denote the space $B C(\mathbb{R})$ endowed with the norm $\|\cdot\|_{\rho}$. As it is easy to check, $B C_{\rho}(\mathbb{R})$ is a Banach space.

Let us define the linear operator $\mathcal{S}$ in $B C_{\rho}(\mathbb{R})$ by

$$
\mathcal{S}(h)(t)=\frac{1}{\alpha_{2}-\alpha_{1}} u_{h}(t), \quad t \in \mathbb{R}
$$

where $u_{h}$ was defined in (2.1).

By virtue of property iii) of Lemma 2.1 , if $h(t) \in[-\beta, 0]$ for every $t \in \mathbb{R}$, then $0 \leq S(h)(t) \leq 1$ for every $t \in \mathbb{R}$. Moreover, from the linearity of $\mathcal{S}$ and property $i i)$ of the same Lemma we get that $\mathcal{S}$ is monotone decreasing with respect the partial ordering in $B C(\mathbb{R})$ induced by the cone $K:=\{h \in$ $B C(\mathbb{R}): h(t) \geq 0$ for every $t \in \mathbb{R}\}$, i.e.

$$
h_{1}(t) \leq h_{2}(t) \text { for every } t \in \mathbb{R} \quad \Rightarrow \quad S\left(h_{1}\right)(t) \geq S\left(h_{2}(t)\right) \text { for every } t \in \mathbb{R} .
$$

Finally, from property $i$ ) of Lemma 2.1, it follows that $S(h)$ is a solution of the following second order linear differential equation:

$$
u^{\prime \prime}(t)+c u^{\prime}(t)-\beta u(t)=h(t), \quad \text { a.e. } t \in \mathbb{R} .
$$


The next Lemma states that $\mathcal{S}$ is continuous with respect to the norm $\|\cdot\|_{\rho}$ for $\rho>0$ small enough.

Lemma 2.2. The operator $\mathcal{S}$ is continuous in $B C_{\rho}(\mathbb{R})$ for every $\rho<\min \left\{-\alpha_{1}, \alpha_{2}\right\}$. More precisely, there exists a constant $k=k(\rho)$ such that

$$
\left\|S\left(h_{1}\right)-S\left(h_{2}\right)\right\|_{\rho} \leq k\left\|h_{1}-h_{2}\right\|_{\rho} \quad \text { for every } h_{1}, h_{2} \in B C(\mathbb{R}) .
$$

Proof. By the linearity of $\mathcal{S}$ it suffices to prove the continuity at the origin. To this aim, notice that

$$
\begin{aligned}
\left|u_{h}(t)\right| & \leq \int_{-\infty}^{t}|h(s)| e^{\alpha_{1}(t-s)} \mathrm{d} s+\int_{t}^{+\infty}|h(s)| e^{\alpha_{2}(t-s)} \mathrm{d} s \\
& =\int_{-\infty}^{t}\left(|h(s)| e^{-\rho|s|}\right) e^{\alpha_{1}(t-s)+\rho|s|} \mathrm{d} s+\int_{t}^{+\infty}\left(|h(s)| e^{-\rho|s|}\right) e^{\alpha_{2}(t-s)+\rho|s|} \mathrm{d} s \\
& \leq \int_{-\infty}^{t}|| h\left\|_{\rho} e^{\alpha_{1}(t-s)+\rho|s|} \mathrm{d} s+\int_{t}^{+\infty}|| h\right\|_{\rho} e^{\alpha_{2}(t-s)+\rho|s|} \mathrm{d} s \\
& =\|h\|_{\rho}\left(\int_{-\infty}^{t} e^{\alpha_{1}(t-s)+\rho|s|} \mathrm{d} s+\int_{t}^{+\infty} e^{\alpha_{2}(t-s)+\rho|s|} \mathrm{d} s\right) .
\end{aligned}
$$

Then,

$$
\left|u_{h}(t)\right| e^{-\rho|t|} \leq\|h\|_{\rho}\left(\int_{-\infty}^{t} e^{\alpha_{1}(t-s)+\rho|s|} \mathrm{d} s+\int_{t}^{+\infty} e^{\alpha_{2}(t-s)+\rho|s|} \mathrm{d} s\right) e^{-\rho|t|} .
$$

We now proceed distinguishing the two cases $t \geq 0$ and $t<0$. If $t \geq 0$, by the upper limitations on $\rho$ we have

$$
\begin{aligned}
& \left(\int_{-\infty}^{t} e^{\alpha_{1}(t-s)+\rho|s|} \mathrm{d} s+\int_{t}^{+\infty} e^{\alpha_{2}(t-s)+\rho|s|} \mathrm{d} s\right) e^{-\rho|t|} \\
= & \left(\int_{-\infty}^{0} e^{-\left(\alpha_{1}+\rho\right) s} \mathrm{~d} s+\int_{0}^{t} e^{\left(\rho-\alpha_{1}\right) s} \mathrm{~d} s\right) e^{\alpha_{1} t-\rho t}+\left(\int_{t}^{+\infty} e^{\left(\rho-\alpha_{2}\right) s} \mathrm{~d} s\right) e^{\alpha_{2} t-\rho t} \\
= & \left(\frac{1}{-\left(\alpha_{1}+\rho\right)}+\frac{e^{\left(\rho-\alpha_{1}\right) t}-1}{\rho-\alpha_{1}}\right) e^{\left(\alpha_{1}-\rho\right) t}+\left(\frac{e^{\left(\rho-\alpha_{2}\right) t}}{\alpha_{2}-\rho}\right) e^{\left(\alpha_{2}-\rho\right) t} \\
= & \frac{e^{\left(\alpha_{1}-\rho\right) t}}{-\left(\rho+\alpha_{1}\right)}+\frac{1-e^{\left(\alpha_{1}-\rho\right) t}}{\rho-\alpha_{1}}+\frac{1}{\alpha_{2}-\rho} \leq \frac{1}{-\left(\alpha_{1}+\rho\right)}+\frac{1}{\rho-\alpha_{1}}+\frac{1}{\alpha_{2}-\rho}=\frac{2 \alpha_{1}}{\rho^{2}-\alpha_{1}^{2}}+\frac{1}{\alpha_{2}-\rho}
\end{aligned}
$$

since being $\alpha_{1}-\rho<0$ we get $0<e^{\left(\alpha_{1}-\rho\right) t}<1$ for every $t \geq 0$. Therefore, by (2.7) we obtain

$$
\left|u_{h}(t)\right| e^{-\rho|t|} \leq k_{+}\|h\|_{\rho} \quad \text { for every } t \geq 0
$$

where $k_{+}:=\left(\frac{-2 \alpha_{1}}{\alpha_{1}^{2}-\rho^{2}}+\frac{1}{\alpha_{2}-\rho}\right)>0$.

Analogously, if $t<0$ we have

$$
\begin{aligned}
& \left(\int_{-\infty}^{t} e^{\alpha_{1}(t-s)+\rho|s|} \mathrm{d} s+\int_{t}^{+\infty} e^{\alpha_{2}(t-s)+\rho|s|} \mathrm{d} s\right) e^{-\rho|t|} \\
= & \left(\int_{-\infty}^{t} e^{-\left(\alpha_{1}+\rho\right) s} \mathrm{~d} s\right) e^{\left(\alpha_{1}+\rho\right) t}+\left(\int_{t}^{0} e^{-\left(\alpha_{2}+\rho\right) s} \mathrm{~d} s+\int_{0}^{+\infty} e^{\left(\rho-\alpha_{2}\right) s} \mathrm{~d} s\right) e^{\left(\alpha_{2}+\rho\right) t} \\
= & \left(\frac{e^{-\left(\alpha_{1}+\rho\right) t}}{-\left(\alpha_{1}+\rho\right)}\right) e^{\left(\alpha_{1}+\rho\right) t}+\left(-\frac{1-e^{-\left(\alpha_{2}+\rho\right) t}}{\alpha_{2}+\rho}+\frac{1}{\alpha_{2}-\rho}\right) e^{\left(\alpha_{2}+\rho\right) t} \\
= & \frac{1}{-\left(\alpha_{1}+\rho\right)}+\frac{1-e^{\left(\alpha_{2}+\rho\right) t}}{\alpha_{2}+\rho}+\frac{e^{\left(\alpha_{2}+\rho\right) t}}{\alpha_{2}-\rho} \leq \frac{1}{-\left(\alpha_{1}+\rho\right)}+\frac{1}{\alpha_{2}+\rho}+\frac{1}{\alpha_{2}-\rho}=\frac{1}{-\left(\alpha_{1}+\rho\right)}+\frac{2 \alpha_{2}}{\alpha_{2}^{2}-\rho^{2}} .
\end{aligned}
$$


since being $\alpha_{2}+\rho>0$ we get $0<e^{\left(\alpha_{2}+\rho\right) t}<1$ for every $t<0$. Thus, by $(2.7)$

$$
\left|u_{h}(t)\right| e^{-\rho|t|} \leq k_{-}\|h\|_{\rho} \quad \text { for every } t<0
$$

where $k_{-}:=\left(\frac{1}{-\left(\alpha_{1}+\rho\right)}+\frac{2 \alpha_{2}}{\alpha_{2}^{2}-\rho^{2}}\right)$. Hence, setting $k:=\frac{1}{\alpha_{2}-\alpha_{1}} \max \left\{k_{+}, k_{-}\right\}$, by $(2.8),(2.9)$ and the definition of $\mathcal{S}$ we conclude

$$
\|S(h)\|_{\rho} \leq k\|h\|_{\rho} .
$$

In the sequel we will need to consider the operator $\mathcal{S}$ for varying values of $c$. In such situations, we will write $\mathcal{S}_{c}$ to emphasize the dependence on the value $c$. The following proposition concerns the behavior of the operator $\mathcal{S}$ with respect to $c$.

Proposition 2.3. Let $\left(c_{n}\right)_{n}$ be a sequence of numbers converging to some $c^{*} \in \mathbb{R}$. Moreover, let $\left(h_{n}\right)_{n}$ be a sequence in $B C(\mathbb{R})$ of equibounded functions, pointwise convergent to some $h^{*}$. Then, the sequence $\left(\mathcal{S}_{c_{n}}\left(h_{n}\right)\right)_{n}$ pointwise converges to $\mathcal{S}_{c^{*}}\left(h^{*}\right)$.

Proof. Since the sequence $\left(h_{n}\right)_{n}$ is equibounded, then there exists a positive value $K>0$ such that $\left|h_{n}(t)\right| \leq K$ for every $t \in \mathbb{R}$ and $n \in \mathbb{N}$. So, denoted by $\alpha_{1}\left(c_{n}\right)<0, \alpha_{2}\left(c_{n}\right)>0$ the two roots of the algebraic equation $x^{2}+c_{n} x-\beta=0$, we have $\alpha_{1}\left(c_{n}\right) \rightarrow \alpha_{1}\left(c^{*}\right)<0$ and $\alpha_{2}\left(c_{n}\right) \rightarrow \alpha_{2}\left(c^{*}\right)>0$ and by the dominated convergence theorem

$$
\int_{-\infty}^{t} h_{n}(s) e^{-\alpha_{1}\left(c_{n}\right) s} \mathrm{~d} s \rightarrow \int_{-\infty}^{t} h^{*}(s) e^{-\alpha_{1}\left(c^{*}\right) s} \mathrm{~d} s, \quad \int_{t}^{+\infty} h_{n}(s) e^{-\alpha_{2}\left(c_{n}\right) s} \mathrm{~d} s \rightarrow \int_{t}^{+\infty} h^{*}(s) e^{-\alpha_{2}\left(c^{*}\right) s} \mathrm{~d} s
$$

as $n \rightarrow+\infty$. Hence, recalling the definition of the operator $\mathcal{S}_{c}$ we obtain $\mathcal{S}_{c_{n}}\left(h_{n}\right)(t) \rightarrow S_{c^{*}}\left(h^{*}\right)(t)$ for every $t \in \mathbb{R}$.

Our approach for finding heteroclinic solutions is based on the method of super and sub-solutions, which will serve as barriers. The following result states conditions which guarantee that a function $\phi$ is a lower [upper] bound for the operator $\mathcal{S}$.

Lemma 2.4. Let $\phi \in B C(\mathbb{R})$ be given. Assume that there exist $-\infty=\tau_{0}<\tau_{1}<\cdots<\tau_{N}<\tau_{N+1}=+\infty$ such that $\phi \in C^{2}\left(\tau_{i}, \tau_{i+1}\right)$, with $\phi^{\prime}, \phi^{\prime \prime}$ bounded in $\left(\tau_{i}, \tau_{i+1}\right)$, for every $i=0, \ldots, N$.

Moreover, assume that for every $i=0, \ldots, N+1$ there exist finite the limits $\phi^{\prime}\left(\tau_{i}^{-}\right)$and $\phi^{\prime}\left(\tau_{i}^{+}\right)$(of course for $i=0$ just the right limit and for $i=N+1$ just the left one).

Define $h_{0}$ by

$$
h_{0}(t)=\phi^{\prime \prime}(t)+c \phi^{\prime}(t)-\beta \phi(t), \quad \text { for every } t \in \mathbb{R}, t \neq \tau_{i}, i=1, \ldots, N .
$$

Then,

i) $\phi^{\prime}\left(\tau_{i}^{-}\right) \leq \phi^{\prime}\left(\tau_{i}^{+}\right)$for every $i=1, \ldots, N \quad \Rightarrow \quad S\left(h_{0}\right)(t) \geq \phi(t)$ for every $t \in \mathbb{R}$;

ii) $\phi^{\prime}\left(\tau_{i}^{-}\right) \geq \phi^{\prime}\left(\tau_{i}^{+}\right)$for every $i=1, \ldots, N \quad \Rightarrow \quad S\left(h_{0}\right)(t) \leq \phi(t)$ for every $t \in \mathbb{R}$.

Proof. Let us prove statement $i$ ) (the other one being analogous). Fix $t \in \mathbb{R} \backslash\left\{\tau_{i}, i=1, \ldots, n\right\}$ and $k$ such that $t \in\left(\tau_{k}, \tau_{k+1}\right)$. We have

$$
u_{h_{0}}(t)=-e^{\alpha_{1} t} \int_{-\infty}^{t}\left(\phi^{\prime \prime}(s)+c \phi^{\prime}(s)-\beta \phi(s)\right) e^{-\alpha_{1} s} \mathrm{~d} s-e^{\alpha_{2} t} \int_{t}^{+\infty}\left(\phi^{\prime \prime}(s)+c \phi^{\prime}(s)-\beta \phi(s)\right) e^{-\alpha_{2} s} \mathrm{~d} s=
$$


A GENERAL APPROACH FOR FRONT-PROPAGATION IN FUNCTIONAL REACTION-DIFFUSION EQUATIONS 7

$$
\begin{gathered}
=-e^{\alpha_{1} t} \sum_{i=0}^{k-1}\left(\int_{\tau_{i}}^{\tau_{i+1}}\left(\phi^{\prime \prime}(s)+c \phi^{\prime}(s)-\beta \phi(s)\right) e^{-\alpha_{1} s} \mathrm{~d} s\right)-e^{\alpha_{1} t} \int_{\tau_{k}}^{t}\left(\phi^{\prime \prime}(s)+c \phi^{\prime}(s)-\beta \phi(s)\right) e^{-\alpha_{1} s} \mathrm{~d} s \\
-e^{\alpha_{2} t} \int_{t}^{\tau_{k+1}}\left(\phi^{\prime \prime}(s)+c \phi^{\prime}(s)-\beta \phi(s)\right) e^{-\alpha_{2} s} \mathrm{~d} s-e^{\alpha_{2} t} \sum_{i=k+1}^{N}\left(\int_{\tau_{i}}^{\tau_{i+1}}\left(\phi^{\prime \prime}(s)+c \phi^{\prime}(s)-\beta \phi(s)\right) e^{-\alpha_{2} s} \mathrm{~d} s\right) .
\end{gathered}
$$

Integrating by parts on each interval $\left(\tau_{i}, \tau_{i+1}\right)$ and recalling that $\alpha_{1}<0<\alpha_{2}$ are the solutions of the equation $x^{2}+c x-\beta=0$, we get

$$
\begin{gathered}
\int_{\tau_{i}}^{\tau_{i+1}}\left(\phi^{\prime \prime}(s)+c \phi^{\prime}(s)-\beta \phi(s)\right) e^{-\alpha_{1} s} \mathrm{~d} s= \\
=\left(\alpha_{1}+c\right)\left[\phi\left(\tau_{i+1}\right) e^{-\alpha_{1} \tau_{i+1}}-\phi\left(\tau_{i}\right) e^{-\alpha_{1} \tau_{i}}\right]+\phi^{\prime}\left(\tau_{i+1}^{-}\right) e^{-\alpha_{1} \tau_{i+1}}-\phi^{\prime}\left(\tau_{i}^{+}\right) e^{-\alpha_{1} \tau_{i}}
\end{gathered}
$$

for every $i=0, \ldots, k-1$ with the convention $e^{-\alpha_{1}(-\infty)}=0$. Analogously,

$$
\begin{gathered}
\int_{\tau_{i}}^{\tau_{i+1}}\left(\phi^{\prime \prime}(s)+c \phi^{\prime}(s)-\beta \phi(s)\right) e^{-\alpha_{2} s} \mathrm{~d} s= \\
=\left(\alpha_{2}+c\right)\left[\phi\left(\tau_{i+1}\right) e^{-\alpha_{2} \tau_{i+1}}-\phi\left(\tau_{i}\right) e^{-\alpha_{2} \tau_{i}}\right]+\phi^{\prime}\left(\tau_{i+1}^{-}\right) e^{-\alpha_{2} \tau_{i+1}}-\phi^{\prime}\left(\tau_{i}^{+}\right) e^{-\alpha_{2} \tau_{i}}
\end{gathered}
$$

for every $i=k+1, \ldots, N$ with the convention $e^{-\alpha_{2}(+\infty)}=0$. On the other hand,

$$
\int_{\tau_{k}}^{t}\left(\phi^{\prime \prime}(s)+c \phi^{\prime}(s)-\beta \phi(s)\right) e^{-\alpha_{1} s} \mathrm{~d} s=\left(\alpha_{1}+c\right)\left[\phi(t) e^{-\alpha_{1} t}-\phi\left(\tau_{k}\right) e^{-\alpha_{1} \tau_{k}}\right]+\phi^{\prime}(t) e^{-\alpha_{1} t}-\phi^{\prime}\left(\tau_{k}^{+}\right) e^{-\alpha_{1} \tau_{k}}
$$

and analogously,

$\int_{t}^{\tau_{k+1}}\left(\phi^{\prime \prime}(s)+c \phi^{\prime}(s)-\beta \phi(s)\right) e^{-\alpha_{2} s} \mathrm{~d} s=\left(\alpha_{2}+c\right)\left[\phi\left(\tau_{k+1}\right) e^{-\alpha_{2} \tau_{k+1}}-\phi(t) e^{-\alpha_{2} t}\right]+\phi^{\prime}\left(\tau_{k+1}^{-}\right) e^{-\alpha_{2} \tau_{k+1}}-\phi^{\prime}(t) e^{-\alpha_{2} t}$.

Therefore, recalling that $-c=\alpha_{1}+\alpha_{2}$, for every $t \neq \tau_{i}, i=1, \ldots, N$, we get

$$
\begin{aligned}
u_{h_{0}}(t)= & -e^{\alpha_{1} t} \sum_{i=1}^{k}\left(\left(\phi^{\prime}\left(\tau_{i}^{-}\right)-\phi^{\prime}\left(\tau_{i}^{+}\right)\right) e^{-\alpha_{1} \tau_{i}}\right)+\left(\alpha_{2} \phi(t)-\phi^{\prime}(t)\right)+ \\
& -e^{\alpha_{2} t} \sum_{i=k+1}^{N}\left(\left(\phi^{\prime}\left(\tau_{i}^{-}\right)-\phi^{\prime}\left(\tau_{i}^{+}\right)\right) e^{-\alpha_{2} \tau_{i}}\right)-\left(\alpha_{1} \phi(t)-\phi^{\prime}(t)\right) \\
= & -e^{\alpha_{1} t} \sum_{i=1}^{k}\left(\left(\phi^{\prime}\left(\tau_{i}^{-}\right)-\phi^{\prime}\left(\tau_{i}^{+}\right)\right) e^{-\alpha_{1} \tau_{i}}\right)-e^{\alpha_{2} t} \sum_{i=k+1}^{N}\left(\left(\phi^{\prime}\left(\tau_{i}^{-}\right)-\phi^{\prime}\left(\tau_{i}^{+}\right)\right) e^{-\alpha_{2} \tau_{i}}\right)+\left(\alpha_{2}-\alpha_{1}\right) \phi(t) .
\end{aligned}
$$

Since, by assumption, $\phi^{\prime}\left(\tau_{i}^{-}\right) \leq \phi^{\prime}\left(\tau_{i}^{+}\right)$for any $i=1, \ldots, N$, it follows

$S\left(h_{0}\right)(t)-\phi(t)=-\frac{e^{\alpha_{1} t}}{\alpha_{2}-\alpha_{1}} \sum_{i=1}^{k}\left(\left(\phi^{\prime}\left(\tau_{i}^{-}\right)-\phi^{\prime}\left(\tau_{i}^{+}\right)\right) e^{-\alpha_{1} \tau_{i}}\right)-\frac{e^{\alpha_{2} t}}{\alpha_{2}-\alpha_{1}} \sum_{i=k+1}^{N}\left(\left(\phi^{\prime}\left(\tau_{i}^{-}\right)-\phi^{\prime}\left(\tau_{i}^{+}\right)\right) e^{-\alpha_{2} \tau_{i}}\right) \geq 0$

for every $t \neq \tau_{i}, i=1, \ldots, N$. By the continuity of $\phi$ and $u_{h_{0}}$, the statement holds for every $t \in \mathbb{R}$. 


\section{An eXistence Result}

Consider the following functional equation:

$$
u^{\prime \prime}(t)+c u^{\prime}(t)+F(u)(t)=0, \quad t \in \mathbb{R}
$$

where $c>0$, and $F: C(\mathbb{R}) \rightarrow C(\mathbb{R})$ is a given operator.

In the sequel we assume that there exists $\beta>0$ such that the following conditions hold:

(H1) $0 \leq u(t) \leq 1$ for every $t \in \mathbb{R} \quad \Rightarrow \quad 0 \leq F(u)(t)+\beta u(t) \leq \beta$ for every $t \in \mathbb{R}$.

(H2) $u$ monotone decreasing $\Rightarrow F(u)+\beta u$ monotone decreasing.

Fixed a value $\beta>0$ in such a way that conditions (H1) and (H2) hold, define $\mathcal{F}: C(\mathbb{R}) \rightarrow C(\mathbb{R})$ by

$$
\mathcal{F}(u)(t)=-F(u)(t)-\beta u(t), \quad t \in \mathbb{R} .
$$

By using the operator $\mathcal{F}$, equation (3.1) can be equivalently written as follows:

$$
u^{\prime \prime}(t)+c u^{\prime}(t)-\beta u(t)=\mathcal{F}(u)(t), \quad t \in \mathbb{R} .
$$

Setting $M:=\frac{2 \beta}{\sqrt{c^{2}+4 \beta}}$, let us consider the following subset of $B C(\mathbb{R})$ :

$\Omega:=\left\{u \in C(\mathbb{R}): 0 \leq u(t) \leq 1\right.$ for all $t \in \mathbb{R}, u$ is decreasing, $\left|u\left(t_{1}\right)-u\left(t_{2}\right)\right| \leq M\left|t_{1}-t_{2}\right|$ for all $\left.t_{1}, t_{2} \in \mathbb{R}\right\}$.

By assumption (H1), we have $\mathcal{F}(u) \in B C(\mathbb{R})$ for every $u \in \Omega$, so we can define the composition operator

$$
\mathcal{G}(u):=\mathcal{S}(\mathcal{F}(u)), \quad \text { for } u \in \Omega .
$$

Notice that a function $u \in \Omega$ is a fixed point for the operator $\mathcal{G}$ if and only if it is a solution of equation (3.2). Hence the study of the existence of solutions of (3.1) reduces to the proof of the existence of fixed points for the operator $\mathcal{G}$, as we do in the following theorem.

Theorem 3.1. Assume that conditions (H1), (H2) are verified. Moreover, assume that the operator $F: B C_{\rho}(\mathbb{R}) \rightarrow B C_{\rho}(\mathbb{R})$ is continuous for some $0<\rho<\min \left\{-\alpha_{1}, \alpha_{2}\right\}$.

Then, equation (3.1) has a solution in $\Omega$.

Proof. First note that as an immediate consequence of conditions (H1), (H2) and statements iii)- iv) of Lemma 2.1, we have

$$
\mathcal{G}(\Omega) \subseteq \Omega
$$

Moreover, $\mathcal{G}$ is continuous in $\Omega$ with respect to the norm $\|\cdot\|_{\rho}$, indeed if $\left(u_{n}\right)_{n}$ is a sequence in $\Omega$ converging to $u \in \Omega$, then

$$
\left\|\mathcal{G}\left(u_{n}\right)-\mathcal{G}(u)\right\|_{\rho}=\| \mathcal{S}\left(\mathcal{F}\left(u_{n}\right)-\mathcal{S}(\mathcal{F}(u))\left\|_{\rho} \leq k\right\| \mathcal{F}\left(u_{n}\right)-\mathcal{F}(u)\left\|_{\rho} \leq k\right\| F\left(u_{n}\right)-F(u)\left\|_{\rho}+k \beta\right\| u_{n}-u \|_{\rho}\right.
$$

where $k=k(\rho)$ is the constant given by Lemma 2.2. Then, the continuity of $\mathcal{G}$ follows from the continuity of $F$.

Observe now that $\Omega$ is a nonempty, convex subset of the Banach space $B C_{\rho}(\mathbb{R})$, so in order to apply the Schauder fixed point theorem it remains to show that $\Omega$ is compact.

To this purpose, let $\left(u_{n}\right)_{n}$ be a given sequence in $\Omega$. By the definition of $\Omega$ we get that $\left(u_{n}\right)_{n}$ is equibounded and equiuniformly continuous. So, by the Ascoli-Arzelà theorem, its restriction to the interval $I_{1}=[-1,1]$ admits a subsequence $\left(u_{n_{1, k}}\right)_{k}$ uniformly convergent in $I_{1}$ to a function $v_{1}(t)$. Similarly, 
in the interval $I_{2}=[-2,2]$ the sequence $\left(u_{n_{1, k}}\right)_{k}$ admits a further subsequence $\left(u_{n_{2, k}}\right)_{k}$ uniformly convergent in $I_{2}$ to a function $v_{2}(t)$. Of course, $v_{2}(t)=v_{1}(t)$ for $t \in I_{1}$. By induction, for every $m \in \mathbb{N}$ the sequence $\left(u_{n_{m-1, k}}\right)_{k}$ admits a further subsequence $\left(u_{n_{m, k}}\right)_{k}$ uniformly convergent in $I_{m}$ to a function $v_{m}(t)$ satisfying $v_{m}(t)=v_{m-1}(t)$ for $t \in I_{m-1}$.

Consider now the "diagonal" subsequence $\left(u_{n_{k, k}}\right)_{k}$ and the function $v: \mathbb{R} \rightarrow \mathbb{R}$ defined by $v(t)=v_{m}(t)$ if $t \in I_{m}$. Clearly, $v$ is well defined, continuous and decreasing; moreover $0 \leq v(t) \leq 1$ for any $t \in \mathbb{R}$, and $\left|v\left(t_{1}\right)-v\left(t_{2}\right)\right| \leq M\left|t_{1}-t_{2}\right|$ for and any $t_{1}, t_{2} \in \mathbb{R} ;$ that is, $v$ belongs to $\Omega$.

Finally, fix $\varepsilon>0$ and choose $L \in \mathbb{N}$ such that $e^{-\rho|t|}<\frac{\varepsilon}{2}$ for any $t$ with $|t|>L$. We have

$$
\left|u_{n_{k, k}}(t)-v(t)\right| e^{-\rho|t|} \leq 2 e^{-\rho|t|}<\varepsilon,
$$

for any $t$ with $|t|>L$. On the other hand, since $\left\{u_{n_{L, k}}(t)\right\}$ converges to $v_{L}(t)=v(t)$ uniformly on $I_{L}$, and since $\left\{u_{n_{k, k}}\right\}$ is a subsequence of $\left\{u_{n_{L, k}}(t)\right\}$, we have

$$
\sup _{t \in[-L, L]}\left|u_{n_{k, k}}(t)-v(t)\right| \rightarrow 0, \quad k \rightarrow \infty .
$$

Hence, there exists $\bar{k}>L$ such that for any $k>\bar{k}$ one has

$$
\left|u_{n_{k, k}}(t)-v(t)\right| e^{-\rho|t|} \leq\left|u_{n_{k, k}}(t)-v(t)\right|<\varepsilon \quad \text { for every } t \in[-L, L] .
$$

Consequently, taking (3.3) into account we get

$$
\left\|u_{n_{k, k}}-v\right\|_{\rho}<\varepsilon \quad \text { for every } k>\bar{k}
$$

i.e. the sub-sequence $\left(u_{n_{k, k}}\right)_{k}$ converges to $v$ with respect to the norm $\|\cdot\|_{\rho}$ and then $\Omega$ is compact.

By applying the Schauder fixed point Theorem we achieve the existence of a fixed point for the operator $\mathcal{G}$ and this concludes the proof.

The following Proposition concerning the asymptotical properties of the decreasing solutions of equation (3.1), will be used in the sequel.

Proposition 3.2. Assume that conditions (H1), (H2) are verified. Moreover, assume that the operator $F: B C_{\rho}(\mathbb{R}) \rightarrow B C_{\rho}(\mathbb{R})$ is continuous for some $0<\rho<\min \left\{-\alpha_{1}, \alpha_{2}\right\}$ and has constant sign, i.e.

$F(u)(t) \leq 0($ or $F(u)(t) \geq 0) \quad$ for every decreasing, non-negative $u \in C(\mathbb{R})$, and every $t \in \mathbb{R}$.

Then, if $u \in C^{2}(\mathbb{R})$ is a decreasing solution of equation (3.1), with $0 \leq u(t) \leq 1$, it satisfies

$$
u^{\prime}( \pm \infty)=u^{\prime \prime}( \pm \infty)=0,
$$

and the constant $c$ has the same sign of the operator $F$.

Proof. Fix $\tau<t$. Integrating the equation $(3.1)$ in $(\tau, t)$ we obtain

$$
u^{\prime}(t)=u^{\prime}(\tau)-c(u(t)-u(\tau))-\int_{\tau}^{t} F(u)(s) \mathrm{d} s
$$

and by the assumption (3.4) we deduce the existence in $\mathbb{R} \cup\{ \pm \infty\}$ of the limit $\lim _{t \rightarrow+\infty} u^{\prime}(t)$, which has to be null by the boundedness of the function $u(t)$. Similarly we can prove that $u^{\prime}(-\infty)=0$. Moreover, from the previous equation, taking the limits as $t \rightarrow+\infty, \tau \rightarrow-\infty$ one derives that $c$ has the same sign of $F$. 
Finally, by assumptions (H1)-(H2) we have that $F(u)(t)+\beta u(t)$ is a decreasing bounded function, so there exist in $\mathbb{R}$ the limits $F(u)( \pm \infty)$. Hence, by equation (3.1) there exist in $\mathbb{R}$ also the limits $u^{\prime \prime}( \pm \infty)$, which have to be null owing the boundedness of $u^{\prime}$ as $|t| \rightarrow+\infty$.

\section{Boundary VAlue PRoblem}

In this section we finally investigate the solvability of the following boundary value problem:

$$
\left\{\begin{array}{l}
u^{\prime \prime}(t)+c u^{\prime}(t)+F(u)(t)=0, \quad \text { a.e. } t \in \mathbb{R} \\
u(-\infty)=1, \quad u(+\infty)=0 .
\end{array}\right.
$$

Note that the solution of equation (3.1) found in the proof of Theorem 3.1 may be trivial, i.e. constant. In order to obtain heteroclinic solutions we need further conditions, such as the existence of suitable super and sub-solutions. To this end, let us now introduce the following definition.

Definition 4.1. A decreasing function $\phi \in B C(\mathbb{R})$ is said to be a super-solution [respectively sub-solution] of (3.1) if there exist $-\infty=\tau_{0}<\tau_{1}<\cdots<\tau_{N}<\tau_{N+1}=+\infty$ such that:

(i) $\phi \in C^{2}\left(\tau_{i}, \tau_{i+1}\right)$, with $\phi^{\prime}, \phi^{\prime \prime}$ bounded, for every $i=0, \ldots, N$;

(ii) the limits $\phi^{\prime}\left(\tau_{i}^{-}\right), \phi^{\prime}\left(\tau_{i}^{+}\right)$exist finite with $\phi^{\prime}\left(\tau_{i}^{-}\right) \geq \phi^{\prime}\left(\tau_{i}^{+}\right)\left[\phi^{\prime}\left(\tau_{i}^{-}\right) \leq \phi^{\prime}\left(\tau_{i}^{+}\right)\right], \quad i=1, \cdots, N$;

(iii) the following differential inequality holds:

$$
\phi^{\prime \prime}(t)+c \phi^{\prime}(t)+F(\phi)(t) \leq 0 \quad\left[\phi^{\prime \prime}(t)+c \phi^{\prime}(t)+F(\phi)(t) \geq 0\right] \quad \text { for every } t \neq \tau_{i}, i=1, \cdots, N .
$$

Remark 4.2. The enlargement of the class of admissible super and sub-solutions to possible non-smooth functions is motivated by the difficulty to find well-ordered smooth super and sub-solutions, due to the lack of monotonicity of $F$ (see [10] for recent comparison results for non-functional equations). In this setting, the relation between the left and right derivatives in the non-smoothness points (condition (ii)) is fundamental. Recently, some papers appeared using a more general definition, without requiring (ii) (see [14], [5], [6], [12], [9]), but the arguments there used do not work (see [15]).

The following theorem provides sufficient conditions for the solvability of problem (4.1).

Theorem 4.3. Assume that there exists $\beta>0$ such that conditions (H1), (H2) are verified. Moreover, assume that the following condition holds:

(H3) $u_{1}(t) \leq u_{2}(t)$ for every $t \in \mathbb{R} \Rightarrow F\left(u_{1}\right)(t)+\beta u_{1}(t) \leq F\left(u_{2}\right)(t)+\beta u_{2}(t)$ for every $t \in \mathbb{R}$.

Fixed $0<\rho<\min \left\{-\alpha_{1}, \alpha_{2}\right\}$, assume that the operator $F: C(\mathbb{R}) \rightarrow C(\mathbb{R})$ is continuous with respect to the norm $\|\cdot\|_{\rho}$.

Finally, assume that there exist a pair $\phi, \psi$ of sub and super-solutions of (3.1) such that $0 \leq \phi(t) \leq$ $\psi(t) \leq 1$ for every $t \in \mathbb{R}$.

Then, the differential equation in (4.1) admits a decreasing solution $u \in \Omega$, such that

$$
\phi(t) \leq u(t) \leq \psi(t) \quad \text { for every } t \in \mathbb{R} .
$$

Therefore, if

$$
\phi(-\infty)=1, \quad \psi(+\infty)=0
$$

then, $u$ solves the boundary value problem (4.1) too. 
Proof. Let $\Omega$ and $\mathcal{G}$ as in Section 3. Consider the following subset of $B C(\mathbb{R})$ :

$$
\hat{\Omega}=\{u \in \Omega: \phi(t) \leq u(t) \leq \psi(t)\} .
$$

Of course, $\hat{\Omega}$ is nonempty and convex. Moreover, since it is a closed subset of $\Omega$, which is compact, also $\hat{\Omega}$ is compact in the Banach space $B C_{\rho}(\mathbb{R})$.

Observe now that $\mathcal{G}(\hat{\Omega}) \subseteq \hat{\Omega}$. Indeed, we already proved that $\mathcal{G}(\Omega) \subseteq \Omega$, so it suffices to show that

$$
\phi(t) \leq \mathcal{G}(u)(t) \leq \psi(t) \quad \text { for every } u \in \hat{\Omega}, t \in \mathbb{R} .
$$

Let us prove that $\mathcal{G}(u)(t) \geq \phi(t)$ for every $t \in \mathbb{R}$ (the other inequality being similar). Notice that condition (H3) implies that $\mathcal{F}(u)(t) \leq \mathcal{F}(\phi)(t)$ for every $t \in \mathbb{R}$. So, defined $\eta: \mathbb{R} \rightarrow \mathbb{R}$ by $\eta(t)=$ $\phi^{\prime \prime}(t)+c \phi^{\prime}(t)-\beta \phi(t)$, a.e. $t \in \mathbb{R}$, we have $\eta \in L^{\infty}(\mathbb{R})$, and $\mathcal{F}(\phi)(t) \leq \eta(t)$ a.e. $t \in \mathbb{R}$ as $\phi$ is a sub-solution. Moreover, $\mathcal{S}(\eta)(t) \geq \phi(t)$ for every $t \in \mathbb{R}$ by Lemma 2.4 .

Consequently, by the monotonicity the of operator $\mathcal{S}$ we get

$$
\mathcal{G}(u)(t)=\mathcal{S}(\mathcal{F}(u))(t) \geq \mathcal{S}(\mathcal{F}(\phi))(t) \geq \mathcal{S}(\eta)(t) \geq \phi(t) \quad \text { for every } t \in \mathbb{R} .
$$

Thus, $\mathcal{G}(\hat{\Omega}) \subseteq \hat{\Omega}$. Moreover, we already proved in Theorem 3.1 that $\mathcal{G}$ is continuous in $\Omega$. So, by applying again the Schauder fixed point theorem it follows that $\mathcal{G}$ has a fixed point $u \in \hat{\Omega}$, which results to be a solution of equation (3.1).

Moreover, if $0 \leq \phi(t) \leq u(t) \leq \psi(t) \leq 1$ for every $t \in \mathbb{R}$, the conditions $\phi(-\infty)=1$ and $\psi(+\infty)=0$ respectively imply that $u(-\infty)=1$ and $u(+\infty)=0$ and, consequently, $u$ is a solution of problem (4.1).

Concerning the properties of the set of the values of the speed $c$ for which problem (4.1) admits solutions, we are able to prove that it is closed provided that the problem is autonomous, in the sense specified by the following definition.

Definition 4.4. We will say that the boundary value problem (4.1) is autonomous, if the following property holds:

$$
u(t) \text { is a solution to }(4.1) \Rightarrow u(t+k) \text { is a solution to (4.1) too, for every } k \in \mathbb{R} .
$$

Proposition 4.5. Let $F: C(\mathbb{R}) \rightarrow C(\mathbb{R})$ be a continuous operator with respect to the norm $\|\cdot\|_{\rho}$, satisfying assumptions (H1)-(H2) and (3.4). Assume that problem (4.1) is autonomous and

$$
\exists \lim _{t \rightarrow \pm \infty} F(u)(t) \neq 0 \quad \text { for every decreasing function } u \in C(\mathbb{R}) \text { such that } u( \pm \infty) \in(0,1) .
$$

Let $\mathcal{C}$ denote the set of the admissible speeds for problem (4.1), i.e.

$$
\mathcal{C}:=\{c>0: \text { problem (4.1) admits a decreasing solution }\} .
$$

Then $\mathcal{C}$ is a closed set (possibly empty).

Proof. As we already observed in the previous section, a decreasing function $u$ is a solution to the differential equation in (4.1) if and only if it is a fixed point for the operator $\mathcal{G}$. Since now the parameter $c$ is not fixed, from now on we use the notation $\mathcal{G}_{c}$ for making explicit the dependence on the speed $c$.

So, assume that $\mathcal{C}$ is nonempty and take a sequence $\left(c_{n}\right)_{n}$ in $\mathcal{C}$ converging to a value $c^{*}$. Let $u_{n}(t)$ denote a decreasing solution to problem (4.1) for $c=c_{n}$, satisfying $u_{n}(0)=\frac{1}{2}$ (this is possible since the problem is autonomous). By Lemma 2.1, part iii), we deduce that $\left|u_{n}^{\prime}(t)\right| \leq \frac{2 \beta}{\sqrt{c_{n}^{2}+4 \beta}} \leq \sqrt{\beta}$ for every 
$t \in \mathbb{R}$. Hence, by the same argument used in the proof of Theorem 3.1 for proving that $\Omega$ is compact, one can show that there exists a sub-sequence, again denoted $\left(u_{n}\right)_{n}$, uniformly converging in every compact set to a decreasing function $u^{*}$.

By the continuity of operator $F$ and assumption $(\mathrm{H} 1)$, we get that $\left(\mathcal{F}\left(u_{n}\right)\right)_{n}$ is an equibounded sequence pointwise convergent to $\mathcal{F}\left(u^{*}\right)$. So, by Proposition 2.3 we deduce

$$
\mathcal{G}_{c^{*}}\left(u^{*}\right)(t)=\lim _{n \rightarrow+\infty} \mathcal{G}_{c_{n}}\left(u_{n}\right)(t)=\lim _{n \rightarrow+\infty} u_{n}(t)=u^{*}(t) .
$$

Therefore, the function $u^{*}$ is a fixed point for the operator $\mathcal{G}_{c^{*}}$ and this means that it is a solution of the differential equation in (4.1) for $c=c^{*}$. Moreover, $u^{*}(0)=\frac{1}{2}$, so $u^{*}$ is not one of the trivial solutions $u(t) \equiv 0$ or $u(t) \equiv 1$.

Let us denote by $\ell^{-}:=u(-\infty) \geq \frac{1}{2}$ and $\ell^{+}:=u(+\infty) \leq \frac{1}{2}$. Since by Proposition 3.2 we have $u^{\prime}(-\infty)=u^{\prime \prime}(-\infty)=0$, then by assumption (4.3) we deduce $\ell^{-}=1$ and $\ell^{+}=0$. Hence $u^{*}$ is a solution to problem (4.1) for $c=c^{*}$.

\section{Applichtions and examples}

In this section we present some non-local reaction-diffusion equations which can be handled by means of the approach we introduced here. More in detail, as mentioned in Introduction, we refer to models whose reaction term has a retarded component or depends on a convolution integral.

\section{5.a - Reaction-diffusion equations with delay}

Fixed $T^{*}>0$, let $f: C\left(\left[-T^{*}, 0\right]\right) \rightarrow[0,+\infty)$ be a given continuous operator (with respect to the usual topology in $\left.C\left(\left[-T^{*}, 0\right]\right)\right)$. Let us consider the following partial differential equation

$$
v_{\tau}=v_{x x}+f\left(v^{\tau}(x)\right)
$$

where $v^{\tau}(x) \in C\left(\left[-T^{*}, 0\right]\right)$ is the function defined by $v^{\tau}(x)(\theta):=v(\tau+\theta, x)$, for $\theta \in\left[-T^{*}, 0\right]$.

In the sequel we will assume that the constant functions 0 and 1 are stationary states for the equation (D), that is

$$
f(\mathbf{1})=f(\mathbf{0})=0
$$

(here and later on, $\mathbf{k}$ denotes the constant function $w(\theta) \equiv k, \theta \in\left[-T^{*}, 0\right]$ ).

When searching for traveling wave solutions connecting the equilibria 0 and 1 , put $t:=x-c \tau$, $u(t)=u(x-c \tau)$ and consider the functional boundary value problem

$$
\left\{\begin{array}{l}
u^{\prime \prime}(t)+c u^{\prime}(t)+f\left(u_{t, c}\right)=0 \\
u(-\infty)=1, u(+\infty)=0
\end{array}\right.
$$

where $u_{t, c} \in C\left(\left[-T^{*}, 0\right]\right)$ is the function defined by

$$
u_{t, c}(\theta):=u(t-c \theta) \text {. }
$$

In order to treat such a problem by means of the approach presented here, we deal with reaction terms having the following structure:

$$
f(w)=f_{1}(w) \cdot f_{2}(w(0))
$$

where $f_{1}: C\left(\left[-T^{*}, 0\right]\right) \rightarrow[0,+\infty), f_{2}: \mathbb{R} \rightarrow[0,+\infty)$ are continuous functions satisfying the following conditions. 
$(\mathbf{F} 1-\mathbf{A}): \quad f_{1}(\mathbf{0})=0, f_{1}(\mathbf{k})>0 \quad$ for every $k \neq 0$

(F1-B): $\quad f_{1}$ is increasing, that is $f_{1}(u) \leq f_{1}(v)$ whenever $u(\theta) \leq v(\theta)$ for every $\theta \in\left[-T^{*}, 0\right]$;

(F2-A): $\quad f_{2}(1)=0, f_{2}(s)>0$ for every $s \in[0,1)$;

(F2-B): $\quad f_{2}$ is Lipschitzian with Lip-constant $L$.

For every $c>0$ let $F_{c}: C(\mathbb{R}) \rightarrow C(\mathbb{R})$ denote the operator defined by

$$
F_{c}(u)(t):=f\left(u_{t, c}\right)=f_{1}(u(t-c \theta)) f_{2}(u(t)) .
$$

Of course, the operator $F_{c}$ is continuous (with respect to the norm $\|\cdot\|_{\rho}$ ).

The following Lemma concerns the applicability of the method presented in the previous sections.

Lemma 5.1. Assume that the operator $f$ satisfies the properties listed above. Then for every $c>0$ the operator $F_{c}$ satisfies assumptions (H1)-(H3) with the constant $\beta:=L f_{1}(\mathbf{1})>0$.

Proof. As for property (H1), since $f(w) \geq 0$ for every $w \in C\left(\left[-T^{*}, 0\right]\right)$, of course $F_{c}(u)(t)+\beta u(t) \geq 0$ for every $u \in C(\mathbb{R})$. Moreover, by (F2-A) and (F2-B) we have $f_{2}(s) \leq L(1-s)$ for every $s \in[0,1]$, so being $f_{1}\left(u_{t, c}\right) \leq f_{1}(\mathbf{1})$ by $(\mathrm{F} 1-\mathrm{B})$, we deduce $f_{1}\left(u_{t, c}\right) f_{2}(u(t)) \leq \beta(1-u(t))$, that is $F_{c}(u)(t)+\beta u(t) \leq \beta$.

If $u$ is monotone decreasing, then fixed $t_{1}<t_{2}$ we have $u\left(t_{1}-c \theta\right) \geq u\left(t_{2}-c \theta\right)$ for every $\theta \in\left[-T^{*}, 0\right]$, that is $u_{t_{1}, c}(\theta) \geq u_{t_{2}, c}(\theta)$ for every $\theta \in\left[-T^{*}, 0\right]$. Hence, by assumption (F1-B) we get $f_{1}\left(u_{t_{1}, c}\right) \geq f_{1}\left(u_{t_{2}, c}\right)$. Moreover, by $(\mathrm{F} 2-\mathrm{B})$ we have $f_{2}\left(u\left(t_{1}\right)\right) \geq f_{2}\left(u\left(t_{2}\right)\right)-L\left[u\left(t_{1}\right)-u\left(t_{2}\right)\right]$, so

$$
f_{1}\left(u_{t_{1}, c}\right) f_{2}\left(u\left(t_{1}\right)\right) \geq f_{1}\left(u_{t_{2}, c}\right) f_{2}\left(u\left(t_{2}\right)\right)-L f_{1}\left(u_{t_{2}, c}\right)\left[u\left(t_{1}\right)-u\left(t_{2}\right)\right] \geq f_{1}\left(u_{t_{2}, c}\right) f_{2}\left(u\left(t_{2}\right)\right)-\beta\left[u\left(t_{1}\right)-u\left(t_{2}\right)\right] .
$$

Hence,

$$
f_{1}\left(u_{t_{1}, c}\right) f_{2}\left(u\left(t_{1}\right)\right)+\beta u\left(t_{1}\right) \geq f_{1}\left(u_{t_{2}, c}\right) f_{2}\left(u\left(t_{2}\right)\right)+\beta u\left(t_{2}\right)
$$

i.e. condition (H2).

Finally, if $u, v \in C(\mathbb{R})$ satisfy $u(t) \leq v(t)$ for every $t \in \mathbb{R}$ then $f_{1}\left(u_{t, c}\right) \leq f_{1}\left(v_{t, c}\right)$ and $f_{2}(v(t)) \geq$ $f_{2}(u(t))-L[v(t)-u(t)]$, so

$$
f_{1}\left(v_{t, c}\right) f_{2}(v(t)) \geq f_{1}\left(u_{t, c}\right) f_{2}(u(t))-L f_{1}\left(u_{t, c}\right)[v(t)-u(t)] \geq f_{1}\left(u_{t, c}\right) f_{2}(u(t))-\beta[v(t)-u(t)],
$$

that is $f\left(v_{t, c}\right)+\beta v(t) \geq f\left(u_{t, c}\right)+\beta u(t)$, i.e. condition (H3).

The following existence result shows that a pair of ordered super- and sub-solutions can be found under very mild assumptions on the non-functional term $f_{2}$, provided that the functional term $f_{1}$ depends on a simple discrete delay. Moreover, we also obtain an estimate of the rate of decay as $t \rightarrow+\infty$. In this context, we adopt the notation

$$
u(t) \approx e^{-\lambda t} \Leftrightarrow u(t) e^{\lambda t} \rightarrow \ell \in(0,+\infty) \text { as } t \rightarrow+\infty .
$$


Theorem 5.2. Let

$$
f_{1}\left(w_{t, c}\right):=w(t+c T) \quad \text { for some } T \in\left[0, T^{*}\right],
$$

and let $f_{2}$ be satisfying conditions (F2-A), (F2-B), such that

$$
f_{2}(s) \leq f_{2}(0)(1-s) \quad \text { for every } s \in[0,1] .
$$

Then, for every $c>2 \sqrt{f_{2}(0)}$ there exists a positive value $T_{0}=T_{0}(c)$ such that if $0 \leq T \leq T_{0}$ the boundary value problem (5.2) admits a decreasing solution $u$. Moreover, $u(t) \approx e^{-\lambda t}$ as $t \rightarrow+\infty$, for some $\lambda \leq \frac{1}{2}\left(c-\sqrt{c^{2}-4 f_{2}(0)}\right)$.

Proof. In view of Lemma 5.1 and Theorem 4.3, we only need to find a pair of ordered super and subsolutions. To this aim, put $K:=f_{2}(0)$ and let us consider the function

$$
H(\ell, c, T):=\ell^{2}-c \ell+K e^{-c \ell T}, \quad \text { for } \ell, T \geq 0 ; c>2 \sqrt{K} .
$$

Since $H$ is a continuous function satisfying $H(0, c, T)=K>0$ and $H\left(\frac{c}{2}, c, T\right)=\frac{c^{2}}{4}-\frac{c^{2}}{2}+K e^{-\frac{c^{2}}{2} T} \leq$ $K-\frac{c^{2}}{4}<0$, the set

$$
A_{c, T}:=\left\{\ell \in\left(0, \frac{c}{2}\right): H(\ell, c, T)=0\right\}
$$

is a nonempty closed set. Put

$$
\lambda=\lambda(c, T):=\max A_{c, T} .
$$

As a consequence of the previous definition, for every $c, T$ there exists a positive value $\epsilon=\epsilon(c, T)<\lambda$, such that

$$
H(\lambda+\epsilon, c, T)=(\lambda+\epsilon)^{2}-c(\lambda+\epsilon)+K e^{-c(\lambda+\epsilon) T}<0 .
$$

Now, given $M>1$, consider the function

$$
\phi(t):=\max \left\{0,\left(1-M e^{-\epsilon t}\right) e^{-\lambda t}\right\} .
$$

Let $t^{*}$ denote the positive value such that $M e^{-\epsilon t^{*}}=1$. Observe that $0=\phi^{\prime}\left(t^{*-}\right)<\phi^{\prime}\left(t^{*+}\right)$, moreover if $t<t^{*}$ then $\phi^{\prime}(t)=\phi^{\prime \prime}(t)=0$, so $\phi^{\prime \prime}(t)+c \phi^{\prime}(t)+f\left(\phi_{t, c}\right) \geq 0$. Instead, if $t>t^{*}$ (and $t+c T>t^{*}$ too) then

$$
\phi^{\prime}(t)=-\lambda e^{-\lambda t}+M(\epsilon+\lambda) e^{-(\epsilon+\lambda) t} ; \quad \phi^{\prime \prime}(t)=\lambda^{2} e^{-\lambda t}-M(\epsilon+\lambda)^{2} e^{-(\epsilon+\lambda) t} .
$$

If $L$ denotes the Lipschitz constant for $f_{2}$, put $h:=\frac{L}{K}$ we have

$$
f_{2}(s) \geq K(1-h s) \quad \text { for every } s \in[0,1] .
$$

Then,

$$
\begin{gathered}
\phi^{\prime \prime}(t)+c \phi^{\prime}(t)+f\left(\phi_{t, c}\right) \geq \phi^{\prime \prime}(t)+c \phi^{\prime}(t)+K \phi(t+c T)[1-h \phi(t)]= \\
=e^{-\lambda t}\left\{\lambda^{2}-M(\epsilon+\lambda)^{2} e^{-\epsilon t}-c \lambda+c M(\epsilon+\lambda) e^{-\epsilon t}+K\left(1-M e^{-\epsilon t} e^{-\epsilon c T}\right) e^{-c \lambda T}\left[1-h\left(1-M e^{-\epsilon t}\right) e^{-\lambda t}\right]\right\} \geq \\
\geq e^{-\lambda t}\left\{\left(\lambda^{2}-c \lambda+K e^{-c \lambda T}\right)-M e^{-\epsilon t}\left[(\epsilon+\lambda)^{2}-c(\epsilon+\lambda)+K e^{-c(\epsilon+\lambda) T}\right]-K h e^{-\lambda t} e^{-\lambda c T}\right\}= \\
=e^{-\lambda t}\left\{H(\lambda, c, T)-M e^{-\epsilon t} H(\lambda+\epsilon, c, T)-K h e^{-\lambda(t+c T)}\right\} \geq M e^{-(\lambda+\epsilon) t}\left\{-H(\lambda+\epsilon, c, T)-\frac{L}{M} e^{-\lambda c T}\right\},
\end{gathered}
$$

since $H(\lambda, c, T)=0$ and $(\lambda-\epsilon) t>0$. By (5.7) we deduce that there exists a positive value $M_{0}$ such that if $M \geq M_{0}$ the last term in the previous chain of inequalities is positive, and this implies that $\phi$ is a sub-solution for every $M \geq M_{0}$. 
In order to find a super-solution, consider the function

$$
\psi(t):=\frac{1}{1+\alpha e^{\lambda t}} \quad \text { with } \alpha>0 \text { and } \lambda=\lambda(c, T) \text { defined in (5.6). }
$$

Of course, $\psi$ is a decreasing function satisfying $\psi(-\infty)=1, \psi(+\infty)=0$.

Observe that

$$
\psi^{\prime}(t)=-\alpha \lambda \frac{e^{\lambda t}}{\left(1+\alpha e^{\lambda t}\right)^{2}}, \quad \psi^{\prime \prime}(t)=\frac{\alpha \lambda^{2} e^{\lambda t}}{\left(1+\alpha e^{\lambda t}\right)^{3}}\left(\alpha e^{\lambda t}-1\right) .
$$

Therefore, by (5.4) we have

$$
\begin{gathered}
\psi^{\prime \prime}(t)+c \psi^{\prime}(t)+f\left(\psi_{t, c}\right) \leq \psi^{\prime \prime}(t)+c \psi^{\prime}(t)+K \psi(t+c T)[1-\psi(t)]= \\
=\frac{\alpha \lambda^{2} e^{\lambda t}\left(\alpha e^{\lambda t}-1\right)}{\left(1+\alpha e^{\lambda t}\right)^{3}}-\frac{\alpha \lambda c e^{\lambda t}}{\left(1+\alpha e^{\lambda t}\right)^{2}}+K \frac{\alpha e^{\lambda t}}{\left(1+\alpha e^{\lambda t}\right)\left(1+\alpha e^{\lambda t} e^{c \lambda T}\right)}= \\
=\frac{\alpha e^{\lambda t}}{\left(1+\alpha e^{\lambda t}\right)^{3}\left(1+\alpha e^{\lambda t} e^{c \lambda T}\right)}\left\{\lambda^{2}\left(\alpha e^{\lambda t}-1\right)\left(1+\alpha e^{\lambda t} e^{c \lambda T}\right)-\lambda c\left(1+\alpha e^{\lambda t}\right)\left(1+\alpha e^{\lambda t} e^{c \lambda T}\right)+K\left(1+\alpha e^{\lambda t}\right)^{2}\right\} .
\end{gathered}
$$

Hence, putting $A(t):=\frac{\alpha e^{\lambda t}}{\left(1+\alpha e^{\lambda t}\right)^{3}\left(1+\alpha e^{\lambda t} e^{c \lambda T}\right)}>0$ and recalling that $\lambda^{2}-c \lambda+K e^{-c \lambda T}=0$, the last term in the previous chain of equalities becomes

$$
\begin{gathered}
A(t)\left\{\alpha^{2} e^{2 \lambda t} e^{c \lambda T}\left(\lambda^{2}-c \lambda+K e^{-c \lambda T}\right)+\alpha e^{\lambda t}\left[\lambda^{2}-c \lambda+e^{c \lambda T}\left(-\lambda^{2}-c \lambda+2 K e^{-c \lambda T}\right)\right]+\left(K-c \lambda-\lambda^{2}\right)\right\}= \\
=A(t)\left\{-\alpha e^{\lambda t}\left[K e^{-c \lambda T}+e^{c \lambda T}\left(2 \lambda c-3 K e^{-c \lambda T}\right)\right]-\left(2 c \lambda-K-K e^{-c \lambda T}\right)\right\} \cdot
\end{gathered}
$$

Let us now consider the function $h(c):=c \lambda(c, 0)=\frac{c}{2}\left(c-\sqrt{c^{2}-4 K}\right)$, for $c>2 \sqrt{K}$. Observe that $h(c)$ is a strictly decreasing function, indeed

$h^{\prime}(c)=\frac{1}{2}\left(c-\sqrt{c^{2}-4 K}\right)+\frac{c}{2}\left(1-\frac{c}{\sqrt{c^{2}-4 K}}\right)=\frac{1}{2}\left[2 c-\frac{c^{2}}{\sqrt{c^{2}-4 K}}-\sqrt{c^{2}-4 K}\right]=\frac{c \sqrt{c^{2}-4 K}-c^{2}+2 K}{\sqrt{c^{2}-4 K}}<0$.

Then,

So, put

$$
c \cdot \lambda(c, 0)=h(c)>K=\lim _{c \rightarrow+\infty} h(c), \quad \text { for every } c>2 \sqrt{K}
$$

$$
\gamma(c, T):=K e^{-c \lambda T}+e^{c \lambda T}\left(2 \lambda c-3 K e^{-c \lambda T}\right), \quad \delta(c, T):=2 c \lambda-K-K e^{-c \lambda T},
$$

by $(5.10)$ we have $\gamma(c, 0)=\delta(c, 0)=2(h(c)-K)>0$. Hence, for every $c>2 \sqrt{K}$ there exists a positive value $T_{0}=T_{0}(c)$ such that $\gamma(c, T), \delta(c, T)>0$ if $T \in\left[0, T_{0}\right]$. Therefore, for such values of $T$ we get that the term in (5.9) is negative for every $t \in \mathbb{R}$ and this means that $\psi$ is a super-solution.

Let us now show that if we take $\alpha<1-\frac{1}{M}$, then $\phi(t)<\psi(t)$ for every $t \in \mathbb{R}$. Such a relation is trivial for $t \leq t^{*}$, whereas for every $t \geq t^{*}$, since $e^{-\lambda t}<e^{-\epsilon t} \leq e^{-\epsilon t^{*}}=\frac{1}{M}$, we have

$$
\left(1-M e^{-\epsilon t}\right)\left(e^{-\lambda t}+\alpha\right) \leq e^{-\lambda t}+\alpha \leq \frac{1}{M}+\alpha<1,
$$

hence $\phi(t)=\left(1-M e^{-\epsilon t}\right) e^{-\lambda t}<\frac{1}{1+\alpha e^{\lambda t}}=\psi(t)$ for every $t \geq t^{*}$.

Therefore, by applying Theorem 4.3 we deduce that the differential equation in (5.2) admits a decreasing solution $u$ satisfying $\phi(t) \leq u(t) \leq \psi(t)$ for every $t \in \mathbb{R}$. This immediately implies that $u(+\infty)=0$, so it remains to show that $u(-\infty)=1$.

In order to do this, observe that by Proposition 3.2 we have $u^{\prime}(-\infty)=u^{\prime \prime}(-\infty)=0$, so also $\lim _{n \rightarrow+\infty} f\left(u_{-n, c}\right)=0$. Put $\ell:=u(-\infty)$, it is easy to see that the sequence of function $\left(u_{-n, c}(\theta)\right)_{n}$ uniformly converges to the constant function $u(\theta) \equiv \ell$. Indeed, for every fixed $\epsilon>0$, let $\tilde{t}_{\epsilon}$ be such that 
$|u(t)-\ell|<\epsilon$ for every $t<\tilde{t}_{\epsilon}$. So, if we take $\bar{n}=\bar{n}_{\epsilon}>c T^{*}-\tilde{t}_{\epsilon}$ then for every $n \geq \bar{n}_{\epsilon}$ and $\theta \in\left[-T^{*}, 0\right]$ we have $-n-c \theta \leq-\bar{n}_{\epsilon}+c T^{*}<\tilde{t}_{\epsilon}$, so

$$
\left|u_{-n, c}(\theta)-\ell\right|=|u(-n-c \theta)-\ell|<\epsilon \quad \text { for every } \theta \in\left[-T^{*}, 0\right], n \geq \bar{n}_{\epsilon} .
$$

Thus, by the continuity of $f$ we get $f(\ell)=0$ and being $\ell>0$, by assumptions (F1-A) and (F2-A) we deduce $\ell=1$.

Finally, as regards the rate of decay, since $\phi(t), \psi(t) \approx e^{-\lambda t}$ as $t \rightarrow+\infty$, also $u(t)$ does. Moreover, since $H(\lambda(c, 0), c, T)<0$, we have $\lambda \leq \lambda(c, 0)=\frac{1}{2}\left(c-\sqrt{c^{2}-4 K}\right)$.

In the previous theorem we fixed a generic speed $c>2 \sqrt{K}$ and show that if the delay $T$ is sufficiently small there exists a front having speed $c$. In the following result we change point of view, indeed we show that fixed a delay $T$ there exists a bounded interval such that if the speed $c$ belongs to it then there exists a traveling wave having speed $c$.

Theorem 5.3. Under the same assumption of Theorem 5.2, for every $0<T<\frac{1}{f_{2}(0)} \log \frac{4}{3}$ there exists a value $c^{*}=c^{*}(T)>2 \sqrt{f_{2}(0)}$ such that for every $c \in\left[2 \sqrt{f_{2}(0)}, c^{*}\right]$ the boundary value problem (5.2) admits a decreasing solution $u$. Moreover, $u(t) \approx e^{-\lambda t}$ as $t \rightarrow+\infty$ (see (5.3)), where $\lambda \leq \frac{1}{2}\left(c-\sqrt{c^{2}-4 f_{2}(0)}\right)$.

Moreover, $c^{*}(T) \rightarrow+\infty$ as $T \rightarrow 0$.

Proof. Put, as above, $K:=f_{2}(0)$, and considered the function $H(\ell, c, t)$ defined in (5.5), observe that $H\left(\frac{\sqrt{K}}{2}, 2 \sqrt{K}, T\right)=\frac{K}{4}-K+K e^{-K T}=K\left(e^{-K T}-\frac{3}{4}\right)>0$, due to the assumption on the upper limitation of $T$. Then, $\lambda(2 \sqrt{K}, T)>\frac{\sqrt{K}}{2}$, i.e. $2 \sqrt{K} \cdot \lambda(2 \sqrt{K}, T)>K$. Therefore, there exists a value $c^{*}=c^{*}(T)>$ $2 \sqrt{K}$ such that

$$
c \cdot \lambda(c, T)>K \quad \text { for every } \quad c \in\left(2 \sqrt{K}, c^{*}\right) .
$$

The proof of the present result proceeds as that of Theorem 5.2 until formula (5.9). From there on, observe that by (5.11) we get

$$
K e^{-c \lambda T}+e^{c \lambda T}\left(2 c \lambda-3 K e^{-c \lambda T}\right)>K e^{-c \lambda T}+2 K e^{c \lambda T}-3 K=K e^{-c \lambda T}\left(1+2 e^{2 c \lambda T}-3 e^{c \lambda T}\right)>0
$$

and $2 c \lambda-K e^{-c \lambda T}-K>K\left(1-e^{-c \lambda T}\right)>0$, implying again that $\psi$ is a super-solution. Hence, from now on the proof proceeds as that of the previous theorem.

The assertion for $c=2 \sqrt{K}$ and $c=c^{*}$ is a consequence of the closure of the range of the admissible speeds proved in Proposition 4.5.

Finally, as regards the behavior of $c^{*}$ for $T$ small, observe that put

$$
c^{* *}(T):=\sup \{c: c \lambda(c, T)>K\}
$$

this is a continuous function of $T$ taking value on $\mathbb{R} \cup\{+\infty\}$, such that $c^{* *}(0)=+\infty$, by virtue of (5.10). So, the assertion follows.

We present now just an example of applications of the results in this section.

Example 5.4. Let us consider the delayed reaction-diffusion equation

$$
v_{\tau}(\tau, x)=v_{x x}(\tau, x)+K v(\tau-T, x)(1-v(\tau, x))^{p}, \quad \text { with } p \geq 1 .
$$

Put

$$
f(w):=K w(-T)(1-w(0))^{p}, \quad \text { for } w \in C([-T, 0],
$$


by what we just observed in the previous remark, we can apply Theorems 5.2 and 5.3 to deduce the existence of travelling fronts.

\section{5.b - Non-functional Fisher-KPP equations}

Despite the present research is motivated by the study of non-local reaction-diffusion equations, we wish to show how we can fruitfully treat also the non-functional case by means of our approach.

Let us consider the classical equation

$$
u^{\prime \prime}+c u^{\prime}+f(u)=0
$$

where $f:[0,1] \rightarrow \mathbb{R}$ is a Lipschitzian Fisher-type term, that is satisfying $f(u)>0$ in $(0,1), f(0)=f(1)=$ 0 . We extend the definition of $f$ in all $\mathbb{R}$ as the null function and define $F: C(\mathbb{R}) \rightarrow C(\mathbb{R})$ by

$$
F(u)(t)=f(u(t)), \quad t \in \mathbb{R} .
$$

Notice that $F$ is a continuous operator with respect the norm $\|\cdot\|_{\rho}$, for every $\rho>0$, since $f$ is a continuous function.

Set $\beta:=\sup _{u \neq v}\left|\frac{f(u)-f(v)}{u-v}\right|$ the Lipschitz constant of $f$, it is immediate to verify that the operator $F$ satisfies the assumptions $(H 1)-(H 3)$. Moreover, as an application of Theorem 4.3 , we can derive the following result, which is well-known.

Proposition 5.5. Let $f$ be a function as above, differentiable in a right neighborhood of 0 with $f^{\prime}(0)>0$, such that there exist $f^{\prime \prime}(0)>-\infty$ and

$$
0<f(u) \leq f^{\prime}(0) u \quad \text { for every } u \in(0,1) .
$$

Then, for every $c \geq 2 \sqrt{f^{\prime}(0)}$ there exists a decreasing solution $u$ of the problem

$$
\left\{\begin{array}{l}
u^{\prime \prime}+c u^{\prime}+f(u)=0 \\
u(-\infty)=1, u(+\infty)=0
\end{array}\right.
$$

Moreover, $u(t) \approx e^{-\lambda t}$ as $t \rightarrow+\infty$ (see (5.3)), where $\lambda=\frac{1}{2}\left(c-\sqrt{c^{2}-4 f^{\prime}(0)}\right)$.

Proof. Since $f^{\prime \prime}(0)>-\infty$, then there exists positive values $\nu, \delta>0$ such that $f^{\prime}(u) \geq f^{\prime}(0)-\nu u$ for every $u \in[0, \delta)$, with $f^{\prime}(0)>0$ owing to assumption (5.13). So, integrating, we deduce

$$
f(u) \geq f^{\prime}(0) u-\frac{\nu}{2} u^{2}=f^{\prime}(0) u\left(1-\frac{\nu}{2 f^{\prime}(0)} u\right) \quad \text { for every } u \in[0, \delta) .
$$

Therefore, considered the function $\phi(t):=\delta \max \left\{0,\left(1-M e^{-\epsilon t}\right) e^{-\lambda t}\right\} \leq \delta\left(\right.$ with $\left.\lambda=\frac{1}{2}\left(c-\sqrt{c^{2}-4 f^{\prime}(0)}\right)\right)$, by means of the same proof of Theorem 5.2 (rewritten for $T=0$ ), one deduce that the function $\phi$ is a sub-solution.

Moreover, let us consider now the function $\psi(t):=\min \left\{1, e^{-\lambda t}\right\}$. Observe that $0=\psi^{\prime}\left(0^{-}\right)>\psi^{\prime}\left(0^{+}\right)$ and since $f(1)=0$, it is immediate to verify that $\psi^{\prime \prime}(t)+c \psi^{\prime}(t)+f(\psi(t))=0$ for every $t<0$. Instead, if $t>0$ then

$$
\psi^{\prime \prime}(t)+c \psi^{\prime}(t)+f(\psi(t)) \leq \psi^{\prime \prime}(t)+c \psi^{\prime}(t)+f^{\prime}(0) \psi(t)=e^{-\lambda t}\left(\lambda^{2}-c \lambda+f^{\prime}(0)\right)=0 .
$$

Therefore, $\psi$ is a super-solution. Finally, one can easily verify that $\phi(t)<\psi(t)$ for every $t \in \mathbb{R}$. Hence, by applying Theorem 4.3 we deduce the existence of a decreasing solution $u(t)$ satisfying $\phi(t) \leq u(t) \leq \psi(t)$ 
for every $t \in \mathbb{R}$, implying $u(+\infty)=0$ with $u \approx e^{-\lambda t}$ as $t \rightarrow+\infty$. Being $f(u)>0$ for every $u \in(0,1)$ and applying Proposition 3.2, we necessarily have $u(-\infty)=1$ and this concludes the proof.

\section{5.c - Reaction-diffusion equations with convolution integrals}

Let us consider the non-local reaction-diffusion equation

$$
v_{\tau}(\tau, x)=v_{x x}(\tau, x)+f_{0}(v(\tau, x)) \int_{-\infty}^{+\infty} \Phi(x-\sigma) v(\tau, \sigma) \mathrm{d} \sigma,
$$

where $\Phi: \mathbb{R} \rightarrow \mathbb{R}$ is a continuous, non-negative map satisfying

$$
\int_{-\infty}^{+\infty} \Phi(s) \mathrm{d} s=1
$$

and $f_{0}: \mathbb{R} \rightarrow[0,+\infty)$ is a continuous function satisfying the following conditions:

(F0-A): $\quad f_{0}(1)=0, f_{0}(s)>0$ for every $s \in[0,1)$;

(F0-B): $\quad f_{0}$ is Lipschitzian with Lip-constant $L$.

When searching for traveling wave solutions $v(\tau, x)=u(x-c \tau)$, the change of variable $t=x-c \tau$ leads to consider the functional boundary value problem (recall that the convolution product is commutative)

$$
\left\{\begin{array}{l}
u^{\prime \prime}(t)+c u^{\prime}(t)+\left(\int_{-\infty}^{+\infty} \Phi(s) u(t-s) \mathrm{d} s\right) f_{0}(u(t))=0 \\
u(-\infty)=1, u(+\infty)=0 .
\end{array}\right.
$$

In order to treat such a problem by means of the approach presented here, define $F: C(\mathbb{R}) \rightarrow C(\mathbb{R})$ by

$$
F(u)(t)=\left(\int_{-\infty}^{+\infty} \Phi(s) u(t-s) \mathrm{d} s\right) f_{0}(u(t)), \quad t \in \mathbb{R} .
$$

Notice that $F$ is a continuous operator.

The following Lemma concerns the applicability of the method presented in the previous sections.

Lemma 5.6. Assume that the function $f_{0}$ satisfies the properties listed above. Then, the operator $F$ satisfies assumptions (H1)-(H3) with $\beta \geq L$.

Proof. As for property (H1), assume $0 \leq u(t) \leq 1$ for any $t \in \mathbb{R}$. Then, $F(u)(t)+\beta u(t) \geq 0$ for any $t$. Moreover, by (F0-A) and (F0-B) we have $f_{0}(s) \leq L(1-s)$ for every $s \in[0,1]$, so

$$
\left(\int_{-\infty}^{+\infty} \Phi(s) u(t-s) \mathrm{d} s\right) f_{0}(u(t)) \leq L(1-u(t))
$$

and, being $\beta \geq L$, we deduce

$$
-F(u)(t)-\beta u(t)+\beta \geq(\beta-L)(1-u(t)) \geq 0
$$

for any $t$. Hence, condition (H1) holds.

Assume now $u \in C(\mathbb{R})$ monotone decreasing and let us show that $F(u)+\beta u$ is monotone decreasing too. Fixed $t_{1}<t_{2}$ we have $u\left(t_{1}\right) \geq u\left(t_{2}\right)$, so

$$
\int_{-\infty}^{+\infty} \Phi(s) u\left(t_{1}-s\right) \mathrm{d} s \geq \int_{-\infty}^{+\infty} \Phi(s) u\left(t_{2}-s\right) \mathrm{d} s
$$


and by (F0-B) we have $f_{0}\left(u\left(t_{1}\right)\right) \geq f_{0}\left(u\left(t_{2}\right)\right)-L\left(u\left(t_{1}\right)-u\left(t_{2}\right)\right)$. Therefore,

$$
\begin{aligned}
& F(u)\left(t_{2}\right)-F(u)\left(t_{1}\right)=\left(\int_{-\infty}^{+\infty} \Phi(s) u\left(t_{2}-s\right) \mathrm{d} s\right) f_{0}\left(u\left(t_{2}\right)\right)-\left(\int_{-\infty}^{+\infty} \Phi(s) u\left(t_{1}-s\right) \mathrm{d} s\right) f_{0}\left(u\left(t_{1}\right)\right) \leq \\
& \leq\left(\int_{-\infty}^{+\infty} \Phi(s) u\left(t_{2}-s\right) \mathrm{d} s\right)\left[f_{0}\left(u\left(t_{2}\right)\right)-f_{0}\left(u\left(t_{1}\right)\right)\right] \leq \\
& \leq L\left(\int_{-\infty}^{+\infty} \Phi(s) u\left(t_{2}-s\right) \mathrm{d} s\right)\left[u\left(t_{1}\right)-u\left(t_{2}\right)\right] \leq \beta\left[u\left(t_{1}\right)-u\left(t_{2}\right)\right]
\end{aligned}
$$

since $\beta \geq L$. Hence, condition (H2) is satisfied. The proof of the validity of (H3) is analogous.

In order to present a concrete application of our existence result, let us consider the particular function

$$
\Phi_{0}(t)=\frac{b}{2} e^{-b|t|}, \quad t \in \mathbb{R}, \text { for some } b>0 .
$$

The following result states that imposing some further conditions on the function $f_{0}$ and the constants $b$, $c$, a pair of ordered super and sub-solutions can be found and consequently the boundary value problem (5.14) admits solutions.

Theorem 5.7. Let $\Phi_{0}$ be defined by (5.15) and let $f_{0}$ satisfy conditions (F0-A) - (F0-B). Assume that $b>2 \sqrt{f_{0}(0)}$ and

$$
f_{0}(s) \leq f_{0}(0)(1-s) \quad \text { for every } s \in[0,1] .
$$

Then, for every $c \in\left[c_{1}^{*}, c_{2}^{*}\right]$, where

$$
c_{1}^{*}:=\sqrt{2\left(b^{2}-b \sqrt{b^{2}-4 f_{0}(0)}\right)}, \quad c_{2}^{*}:=\sqrt{2\left(b^{2}+b \sqrt{b^{2}-4 f_{0}(0)}\right)},
$$

the boundary value problem (5.14) admits a decreasing solution $u$. Moreover, $u(t) \approx e^{-\lambda t}$ as $t \rightarrow+\infty$ (see (5.3)), for a suitable $\lambda<c / 2$.

Proof. In view of Lemma 5.6 and Theorem 4.3, we only need to find a pair of ordered super and subsolutions.

First of all, observe that it suffices to prove the assertion for $c \in\left(c_{1}^{*}, c_{2}^{*}\right)$. Indeed, in the present framework all the assumptions of Proposition 4.5 are satisfied and then the range of the values of $c$ for which the boundary value problem (5.14) is solvable is closed. So, from now on we fix a constant $c \in\left(c_{1}^{*}, c_{2}^{*}\right)$.

From now on, put $K:=f_{0}(0)$. Moreover, if $L$ denotes the Lipschitz constant of $f_{0}$, put $h:=\frac{L}{K} \geq 1$, we have

$$
f_{0}(s) \geq K(1-h s) \quad \text { for every } s \in[0,1] .
$$

Given $M>1$, consider the function

$$
\phi(t):=\max \left\{0, \frac{1}{h}\left(1-M e^{-\epsilon t}\right) e^{-\lambda t}\right\},
$$

where $0<\epsilon<\lambda$ and $\lambda<\lambda+\epsilon<b$. Let $t^{*}$ denote the positive value such that $M e^{-\epsilon t^{*}}=1$. Observe that $0=\phi^{\prime}\left(t^{*-}\right)<\phi^{\prime}\left(t^{*+}\right)$, moreover if $t<t^{*}$ then $\phi^{\prime}(t)=\phi^{\prime \prime}(t)=0$, and if $t>t^{*}$ then

$$
\phi^{\prime}(t)=-\frac{\lambda}{h} e^{-\lambda t}+\frac{M}{h}(\lambda+\epsilon) e^{-(\lambda+\epsilon) t} ; \quad \phi^{\prime \prime}(t)=\frac{\lambda^{2}}{h} e^{-\lambda t}-\frac{M}{h}(\lambda+\epsilon)^{2} e^{-(\lambda+\epsilon) t} .
$$


To show that $\phi$ is a sub-solution, taking (5.18) into account, we have to prove that

$\phi^{\prime \prime}(t)+c \phi^{\prime}(t)+\left(\int_{-\infty}^{+\infty} \Phi_{0}(s) \phi(t-s) \mathrm{d} s\right) f_{0}(\phi(t)) \geq \phi^{\prime \prime}(t)+c \phi^{\prime}(t)+\left(\int_{-\infty}^{+\infty} \Phi_{0}(s) \phi(t-s) \mathrm{d} s\right) K(1-h \phi(t)) \geq 0$ for every $t$ in $\mathbb{R}$. Now, for $t<t^{*}$ we have

$$
\phi^{\prime \prime}(t)+c \phi^{\prime}(t)+\left(\int_{-\infty}^{+\infty} \Phi_{0}(s) \phi(t-s) \mathrm{d} s\right) K(1-h \phi(t))=K \int_{-\infty}^{+\infty} \Phi_{0}(s) \phi(t-s) \mathrm{d} s \geq 0 .
$$

Instead, for $t>t^{*}$ we have

$$
\begin{gathered}
\phi^{\prime \prime}(t)+c \phi^{\prime}(t)+\left(\int_{-\infty}^{+\infty} \Phi_{0}(s) \phi(t-s) \mathrm{d} s\right) K(1-h \phi(t))= \\
=\frac{e^{-\lambda t}}{h}\left\{\lambda^{2}-M(\lambda+\epsilon)^{2} e^{-\epsilon t}-c \lambda+c M(\lambda+\epsilon) e^{-\epsilon t}+\frac{K b}{2}(1-h \phi(t)) \int_{-\infty}^{t-t^{*}} e^{\lambda s-b|s|}\left(1-M e^{-\epsilon t+\epsilon s}\right) \mathrm{d} s\right\} .
\end{gathered}
$$

Computing the integral in the last formula, and recalling that $M e^{-\epsilon t^{*}}=1$, we obtain

$$
\begin{gathered}
\int_{-\infty}^{t-t^{*}} e^{\lambda s-b|s|}\left(1-M e^{-\epsilon t+\epsilon s}\right) \mathrm{d} s=\int_{-\infty}^{0} e^{\lambda s+b s}\left(1-M e^{-\epsilon t+\epsilon s}\right) \mathrm{d} s+\int_{0}^{t-t^{*}} e^{\lambda s-b s}\left(1-M e^{-\epsilon t+\epsilon s}\right) \mathrm{d} s= \\
=\frac{1}{\lambda+b}-M e^{-\epsilon t} \frac{1}{\lambda+\epsilon+b}+\frac{e^{(\lambda-b)\left(t-t^{*}\right)}-1}{\lambda-b}-M e^{-\epsilon t} \frac{e^{(\lambda+\epsilon-b)\left(t-t^{*}\right)}-1}{\lambda+\epsilon-b}= \\
=\frac{2 b}{b^{2}-\lambda^{2}}-M e^{-\epsilon t} \frac{2 b}{b^{2}-(\lambda+\epsilon)^{2}}+e^{(\lambda-b)\left(t-t^{*}\right)}\left(\frac{1}{b-(\lambda+\epsilon)}-\frac{1}{b-\lambda}\right) .
\end{gathered}
$$

Therefore,

$$
\begin{gathered}
\frac{K b}{2}(1-h \phi(t))\left(\int_{-\infty}^{t-t^{*}} e^{\lambda s-b|s|}\left(1-M e^{-\epsilon t+\epsilon s}\right) \mathrm{d} s\right)= \\
=\frac{K b}{2}\left(1-e^{-\lambda t}+M e^{-\epsilon t-\lambda t}\right)\left(\int_{-\infty}^{t-t^{*}} e^{\lambda s-b|s|}\left(1-M e^{-\epsilon t+\epsilon s}\right) \mathrm{d} s\right)= \\
=\left(1-e^{-\lambda t}+M e^{-\epsilon t-\lambda t}\right)\left(\frac{K b^{2}}{b^{2}-\lambda^{2}}-M e^{-\epsilon t} \frac{K b^{2}}{b^{2}-(\lambda+\epsilon)^{2}}+e^{(\lambda-b)\left(t-t^{*}\right)} \frac{\epsilon K b}{2(b-\lambda)(b-(\lambda+\epsilon))}\right) .
\end{gathered}
$$

Now, since $\lambda<\lambda+\epsilon<b$, we get $(b-\lambda)(b-(\lambda+\epsilon))>0$. Consequently, being $1-h \phi(t) \geq 0$ for every $t$, it follows

$$
\begin{gathered}
\left(1-e^{-\lambda t}+M e^{-\epsilon t-\lambda t}\right)\left(\frac{K b^{2}}{b^{2}-\lambda^{2}}-M e^{-\epsilon t} \frac{K b^{2}}{b^{2}-(\lambda+\epsilon)^{2}}+e^{(\lambda-b)\left(t-t^{*}\right)} \frac{\epsilon K b}{2(b-\lambda)(b-(\lambda+\epsilon))}\right) \geq \\
\geq\left(1-e^{-\lambda t}+M e^{-\epsilon t-\lambda t}\right)\left(\frac{K b^{2}}{b^{2}-\lambda^{2}}-M e^{-\epsilon t} \frac{K b^{2}}{b^{2}-(\lambda+\epsilon)^{2}}\right)= \\
=\frac{K b^{2}}{b^{2}-\lambda^{2}}-M e^{-\epsilon t} \frac{K b^{2}}{b^{2}-(\lambda+\epsilon)^{2}}-e^{-\lambda t}\left(1-M e^{-\epsilon t}\right)\left(\frac{K b^{2}}{b^{2}-\lambda^{2}}-M e^{-\epsilon t} \frac{K b^{2}}{b^{2}-(\lambda+\epsilon)^{2}}\right) .
\end{gathered}
$$

Since $\frac{1}{b^{2}-\lambda^{2}} \leq \frac{1}{b^{2}-(\lambda+\epsilon)^{2}}$ and $0 \leq 1-M e^{-\epsilon t} \leq 1$ for $t>t^{*}$, we have

$$
\begin{gathered}
e^{-\lambda t}\left(1-M e^{-\epsilon t}\right)\left(\frac{K b^{2}}{b^{2}-\lambda^{2}}-M e^{-\epsilon t} \frac{K b^{2}}{b^{2}-(\lambda+\epsilon)^{2}}\right) \leq \\
\leq e^{-\lambda t}\left(1-M e^{-\epsilon t}\right)^{2}\left(\frac{K b^{2}}{b^{2}-(\lambda+\epsilon)^{2}}\right) \leq e^{-\lambda t} \frac{K b^{2}}{b^{2}-(\lambda+\epsilon)^{2}} .
\end{gathered}
$$


Finally,

$$
\begin{gathered}
\left(1-e^{-\lambda t}+M e^{-\epsilon t-\lambda t}\right)\left(\frac{K b^{2}}{b^{2}-\lambda^{2}}-M e^{-\epsilon t} \frac{K b^{2}}{b^{2}-(\lambda+\epsilon)^{2}}+e^{(\lambda-b)\left(t-t^{*}\right)} \frac{\epsilon K b}{2(b-\lambda)(b-(\lambda+\epsilon))}\right) \geq \\
\geq \frac{K b^{2}}{b^{2}-\lambda^{2}}-M e^{-\epsilon t} \frac{K b^{2}}{b^{2}-(\lambda+\epsilon)^{2}}-e^{-\lambda t} \frac{K b^{2}}{b^{2}-(\lambda+\epsilon)^{2}} .
\end{gathered}
$$

Hence, we get

$$
\begin{gathered}
\lambda^{2}-M(\lambda+\epsilon)^{2} e^{-\epsilon t}-c \lambda+c M(\lambda+\epsilon) e^{-\epsilon t}+\frac{K b}{2}(1-h \phi(t))\left(\int_{-\infty}^{t-t^{*}} e^{\lambda s-b|s|}\left(1-M e^{-\epsilon t+\epsilon s}\right) \mathrm{d} s\right) \geq \\
\geq \lambda^{2}-M(\lambda+\epsilon)^{2} e^{-\epsilon t}-c \lambda+c M(\lambda+\epsilon) e^{-\epsilon t}+\frac{K b^{2}}{b^{2}-\lambda^{2}}-M e^{-\epsilon t} \frac{K b^{2}}{b^{2}-(\lambda+\epsilon)^{2}}-e^{-\lambda t} \frac{K b^{2}}{b^{2}-(\lambda+\epsilon)^{2}}= \\
=\lambda^{2}-c \lambda+\frac{K b^{2}}{b^{2}-\lambda^{2}}-M e^{-\epsilon t}\left((\lambda+\epsilon)^{2}-c(\lambda+\epsilon)+\frac{K b^{2}}{b^{2}-(\lambda+\epsilon)^{2}}\right)-e^{-\lambda t} \frac{K b^{2}}{b^{2}-(\lambda+\epsilon)^{2}}= \\
Q(\lambda)-M e^{-\epsilon t} Q(\lambda+\epsilon)-e^{-\lambda t} \frac{K b^{2}}{b^{2}-(\lambda+\epsilon)^{2}},
\end{gathered}
$$

where $Q(s):=s^{2}-c s+\frac{K b^{2}}{b^{2}-s^{2}}$.

We claim that for a suitable choice of $\lambda, \epsilon$ and $M$ the last term in the previous chain of inequalities is non-negative. Indeed, notice that $Q(0)=K$. Moreover, conditions $b>2 \sqrt{K}$ and (5.17) imply that $c<2 b$ and $Q(c / 2)<0$. Consequently, there exist positive numbers $\lambda=\lambda(c, b, K), \epsilon=\epsilon(c, b, K)$ satisfying $\epsilon<\lambda, \lambda+\epsilon<c / 2$, such that

$$
Q(\lambda)=0 \quad \text { and } Q(\lambda+\epsilon)<0
$$

Moreover, since $t>t^{*}>0$, we have $0<e^{-(\lambda-\epsilon) t}<1$, so

$$
\begin{gathered}
Q(\lambda)-M e^{-\epsilon t} Q(\lambda+\epsilon)-e^{-\lambda t} \frac{K b^{2}}{b^{2}-(\lambda+\epsilon)^{2}}=e^{-\epsilon t}\left[-M Q(\lambda+\epsilon)-e^{-(\lambda-\epsilon) t} \frac{K b^{2}}{b^{2}-(\lambda+\epsilon)^{2}}\right] \geq \\
\geq e^{-\epsilon t}\left[-M Q(\lambda+\epsilon)-\frac{K b^{2}}{b^{2}-(\lambda+\epsilon)^{2}}\right] \geq 0
\end{gathered}
$$

provided that $M>0$ is large enough.

Therefore, with the above choice of $\lambda, \epsilon, M=M(b, K, \lambda, \epsilon)=M(c, b, K)$, the function $\phi(t)=$ $\max \left\{0, \frac{1}{h}\left(1-M e^{-\epsilon t}\right) e^{-\lambda t}\right\}$ is a sub-solution.

In order to find a super-solution, consider the function

$$
\psi(t):=\min \left\{1, e^{-\lambda t}\right\}
$$

where the constant $\lambda>0$ is the same as above. Observe that $0=\psi^{\prime}\left(0^{-}\right)>\psi^{\prime}\left(0^{+}\right)$, moreover if $t<0$ then $\psi^{\prime}(t)=\psi^{\prime \prime}(t)=0$, and if $t>0$ then $\psi^{\prime}(t)=-\lambda e^{-\lambda t}, \psi^{\prime \prime}(t)=\lambda^{2} e^{-\lambda t}$.

To show that $\psi$ is a super-solution we have to prove that

$\psi^{\prime \prime}(t)+c \psi^{\prime}(t)+\left(\int_{-\infty}^{+\infty} \Phi_{0}(s) \psi(t-s) \mathrm{d} s\right) f_{0}(\psi(t)) \leq \psi^{\prime \prime}(t)+c \psi^{\prime}(t)+\left(\int_{-\infty}^{+\infty} \Phi_{0}(s) \psi(t-s) \mathrm{d} s\right) K(1-\psi(t)) \leq 0$ for every $t$ in $\mathbb{R}$. Now, for $t<0$ we have $\psi^{\prime \prime}(t)+c \psi^{\prime}(t)+\left(\int_{-\infty}^{+\infty} \Phi_{0}(s) \psi(t-s) \mathrm{d} s\right) K(1-\psi(t))=0$.

Instead, for $t>0$ we have

$\psi^{\prime \prime}(t)+c \psi^{\prime}(t)+\left(\int_{-\infty}^{+\infty} \Phi_{0}(s) \psi(t-s) \mathrm{d} s\right) K(1-\psi(t))=\lambda^{2} e^{-\lambda t}-c \lambda e^{-\lambda t}+K\left(1-e^{-\lambda t}\right) e^{-\lambda t} \int_{-\infty}^{+\infty} \Phi_{0}(s) e^{\lambda s} \mathrm{~d} s=$ 


$$
=e^{-\lambda t}\left(\lambda^{2}-c \lambda+K\left(1-e^{-\lambda t}\right) \int_{-\infty}^{+\infty} \Phi_{0}(s) e^{\lambda s} \mathrm{~d} s\right) .
$$

Since $0 \leq 1-e^{-\lambda t} \leq 1$ for $t>0$, taking account of (5.19), we deduce

$$
\lambda^{2}-c \lambda+K\left(1-e^{-\lambda t}\right) \int_{-\infty}^{+\infty} \Phi_{0}(s) e^{\lambda s} \mathrm{~d} s \leq \lambda^{2}-c \lambda+K \frac{b}{2} \int_{-\infty}^{+\infty} e^{\lambda s-b|s|} \mathrm{d} s=\lambda^{2}-c \lambda+\frac{K b^{2}}{b^{2}-\lambda^{2}}=Q(\lambda)=0 .
$$

Then, the function $\psi(t)$ is a super-solution.

Finally, note that $\phi(t)<\psi(t)$ for every $t \in \mathbb{R}$. In fact, this is trivial for $t \leq t^{*}$, whereas for every $t \geq t^{*}$ we have

$$
\phi(t)=\frac{1}{h}\left(1-M e^{-\epsilon t}\right) e^{-\lambda t}<e^{-\lambda t}=\psi(t) .
$$

Therefore, by applying Theorem 4.3 we deduce that the differential equation in (5.14) admits a decreasing solution $u$ satisfying $\phi(t) \leq u(t) \leq \psi(t)$ for every $t \in \mathbb{R}$. This immediately implies that $u(+\infty)=0$. Finally, by arguing as in the proof of Theorem 5.2, one can prove that $u(-\infty)=1$ and the proof is complete.

Similarly to what we done in the case of delayed equation, we present now an example of application of the previous result.

Example 5.8. Let us consider the non-local reaction-diffusion equation

$$
v_{\tau}(\tau, x)=v_{x x}(\tau, x)+\frac{K b}{2}(1-v(\tau, x))^{p} \int_{-\infty}^{+\infty} e^{-b|x-\sigma|} v(t, \sigma) \mathrm{d} \sigma, \quad \text { with } p \geq 1 .
$$

Put $f_{0}(s):=(1-s)^{p}$, we can apply Theorem 5.7 and deduce the existence of travelling fronts.

Remark 5.9. As we mentioned in Introduction, the classical Fisher-KPP equation with the reaction term $f(u)=K u(1-u)$ can be viewed as a particular case of the equation governed by the convolution integral when the kernel is the Dirac delta function and can be obtained taking the limit as $b \rightarrow+\infty$. Notice that the threshold values $c_{1}^{*}=c_{1}^{*}(b, K), c_{2}^{*}=c_{2}^{*}(b, K)$ given by (5.17) satisfy

$$
\lim _{b \rightarrow+\infty} c_{1}^{*}(b, K)=2 \sqrt{K} \quad \text { and } \quad \lim _{b \rightarrow+\infty} c_{2}^{*}(b, K)=+\infty
$$

in accordance with the circumstance that the classical Fisher-KPP equation in this case admits t.w.s. if and only if $c \geq c^{*}=2 \sqrt{f^{\prime}(0)}=2 \sqrt{K}$.

\section{REFERENCES}

[1] T. Faria - S. Trofimchuk, Nonmonotone travelling waves in a single species reaction-diffusion equation with delay, J. Differ. Equations 228 (2006), 357-376.

[2] P.C. Fife, Mathematical aspects of reacting and diffusing systems, Lecture Notes in Biomathematics 28, SpringerVerlag, Berlin, 1979.

[3] B.H. Gilding - R. Kersner, Travelling Waves in Nonlinear Diffusion-Convection-Reaction, Birkhäuser Verlag: Basel, 2004 .

[4] S.A. Gourley, Travelling front solutions of a nonlocal Fisher equation, J. Math. Biol. 41 (2000), 272-284.

[5] J. Huang - X. Zou, Existence of traveling wavefronts of delayed reaction diffusion systems without monotonicity, Discr. Cont. Dyn. Syst. 9 (4) (2003), 925-936.

[6] J. Huang - X. Zou, Travelling wave solutions in delayed reaction diffusion systems with partial monotonicity, Acta Math. Appl. Sin. 22 (2) (2006), 243-256. 
[7] S. Kamin - P. Rosenau, Convergence to the travelling wave solution for a nonlinear reaction-diffusion equation. , Atti Accad. Naz. Lincei, Cl. Sci. Fis. Mat. Nat., IX. Ser., 15 (3-4) (2004), 271-280.

[8] S. Ma, Traveling wavefronts for delayed reaction-diffusion systems via a fixed point theorem, J. Differ. Equations 171 (2001), 294-314.

[9] S. Ma, Traveling waves for non-local delayed diffusion equations via auxiliary equations, J. Diff. Eqns. 237 (2) (2007), 259-277.

[10] C. Marcelli - F. Papalini, Comparison results and existence of bounded solutions on the whole real line to second order differential equations, preprint.

[11] J.D. Murray, Mathematical Biology, Springer-Verlag: Berlin, 1993.

[12] J. W. So - J. Wu - X. Zou, A reaction-diffusion model for a single species with age structure. I. Travelling-wave fronts on unbounded domains, Proc. R. Soc. Lond. A 457 (2001), 1-13.

[13] A. Volpert - V. Volpert - V. Volpert, Travelling Wave Solutions of Parabolic Systems, Trans. of Math. Monogr. 140, Amer. Math. Soc., Providence, Rhode Island, 1994.

[14] J. Wu - X. Zou, Traveling Wave Fronts of Reaction-Diffusion Systems with Delay, J. Dyn. Diff. Eqns. 13 (3) (2001), 651-687

[15] J. Wu - X. Zou, Erratum: Traveling Wave Fronts of Reaction-Diffusion Systems with Delay, J. Dyn. Diff. Eqns., to appear.

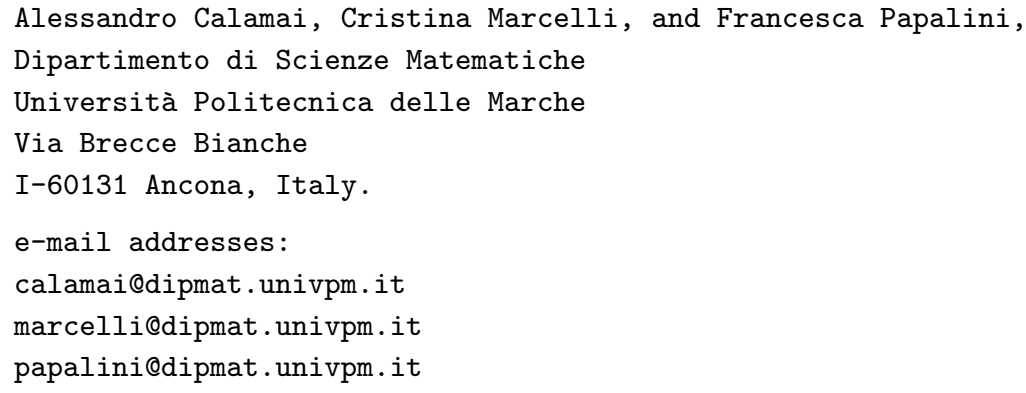

